\title{
THÉORIE DU ROMAN ET THÉORIE DE LA RÉVOLUTION DANS LA PENSÉE DE SARTRE
}

Cristina Diniz Mendonça CREMA ${ }^{1}$

- RÉSUMÉ: En redonnant vie au problème de la transformation des formes tout au long de l'itinéraire de la pensée de Sartre - de la "philosophie pure" à la "monographie historique concrète" -, cette étude essaie de reconstituer quelques moments du processus de gestation de la synthèse sartrienne entre Philosophie, Roman et Révolution. La "monographie historique concrète" aurait-elle chez Sartre la fonction de succédané pour la "Philosophie Traditionnelle" et pour le "Roman Traditionnel"? Ce qui semble conduire l'itinéraire sartrien - de L'être et le néant à L'idiot de la famille -, c'est la recherche d'une forme philosophique, littèraire et historique qui présuppose, sous-jacente, une Théorie du Roman indissolublement liée à une Théorie de la Révolution, plus précisément, à une théorie de la temporalité révolutionnaire qui recèle en elle-même la marque décisive de l'expérience politique de la Guerre, de Occupation et de la Résistance.

- UNITERMES: Théorie du Roman; théorie de la Révolution; "Philosophie Traditionnelle”; Résistance; "apocalypse".

Marx disait que les Allemands ont pensé ce que les Français ont fait, c'est-à-dire qu'ils ont élaboré la théorie d'une révolution qui a eu lieu de l'autre côté du Rhin. Sartre - Un promeneur dans Panis insurgé (titre de sa série de reportages sur l'Insurrection parisienne d'août 1944) - n'a guère eu besoin que de franchir la Seine pour élaborer théoriquement ce que les autres, les Résistants, ont fait dans la pratique politique. Sans avoir besoin de chercher ailleurs, Sartre, à notre avis, a développé la théorie d'un "programme héroique" qui "s'est réalisé point par point sous [ses] yeux", comme il écrit dans l'un de ses sept reportages publiés, au fur et à mesure des événements, dans le journal Combat. Sous les coups de feu des barricades de 1944, une théorie de la révolution éclôt.

En nous interrogeant sur la nature de cette théorie forgée à chaud durant une époque de "haute température historique" (pour utiliser l'expression de Jaurès reprise par Sartre), et en essayant de reconstituer le processus de sa gestation, il nous a paru

1. Departamento de Filosofia - Faculdade de Filosofia e Ciências - UNESP - 17500 - Marília - SP. 
qu'elle tend vers une nouvelle forme de narration (pour ainsi dire) à la fois historique, philosophique et littéraire. Quelle forme?

Si Marx et Engels ont parlé de "décomposition de l'Esprit Absolu" (et désormais il n'est plus possible - si l'on veut "comprendre la réalité humaine dans ce qu'elle a de concret", comme le dit Marcuse dans Culture et Société - de faire de la "Philosophie Traditionnelle"), T. Adorno parle de "décomposition de la forme romanesque" (puisque les conditions sociales n' offrent plus les matériaux adéquats pour la narration - "on ne peut plus narrer, alors que la forme du roman exige la narration" -, il n'est plus possible de faire du "Roman Traditionnel", affirme l'auteur dans Notes sur la Littérature, Flammarion, p. 37 et 41). Il en résulte un double problème pour un certain type de réflexion philosophico-littéraire contemporaine. D'une part, quelle serait la nouvelle façon de conserver "l'unité synthétique", pour employer une expression récurrente chez Sartre, réalisée dans l'Esprit Absolu hégélien? D'autre part, pour revenir à Adorno, comment "rester fidèle" à l' "heritage réaliste" de la forme narrative ("le réalisme lui était immanent") et "suggérer le réel", "dire ce qui existe vraiment"? (Notes su la Littérature, p. 37 et 39). Ou, pour formuler ce dernier problème dans les termes sartriens, comment penser un nouveau "réalisme" qui, tout en préservant la subjectivité, "montre l'homme réel au milieu du monde réel"? (Questions de méthode, p. 37).

On pourrait considérer l'oeuvre sartrienne comme un moment de ce processus de modification historique de la forme philosophique et littéraire. Le problème de la forme de survivance de la philosophie et de la littérature à notre époque fut toujours au centre des préocupations théoriques de Sartre.

Déjà dans Cahiers pour une morale, l'auteur écrit: "Hegel représente un sommet de la philosophie. A partir de lui, régression. Marx apporte ce qu'il n'avait pas donné entièrement [...]. Dégénérescence marxiste ensuite. Dégénérescence allemande post-hégélienne. Heidegger et Husserl petits philosophes. Philosophie française nulle" (p. 67). Plus tard, réfléchissant sur l'ensemble de son propre travail, Sartre fait une affirmation qui pourrait étonner à première vue: "Si, il y a eu deux oeuvres de philosophie pure: $L$ 'Être et le néant et Critique de la raison dialectique, mais c'est un peu en dehors de ce que j'aime faire" (Entretiens avec Michel Sicard, 1977-1978, in Essais sur Sartre, Galilée, p. 380).

Mais si ce que Sartre "aime faire", la vraie nature de son oeuvre, ne se trouve pas dans la "philosophie pure", cela ne se trouve pas non plus dans le "roman traditionnel" (pour reprende l'expression d'Adorno). En 1970, interrogé sur les raisons qui l'auraient conduit à abandonner le roman pour écrire des biographies (le roman serait-il devenu "une forme littéraire impossible"?), l'auteur répond: "Il n'y a plus d'univers naturel du roman et il ne peut plus exister qu'un certain type de roman: le roman "spontané", " "naif' (Situations, IX, dorénavant Sit., p. 122). Et dans un entretien postérieur, Sartre dit que, même en étant fort "fasciné" par le style de Madame Bovary, il sait très bien que l'on ne peut plus écrire comme Flaubert: cette sorte de roman appartient "à un monde qui est un peu passé" (Essais sur Sartre, p. 154). 
Par conséquent, ni "philosophie pure" ni "roman traditionnel" (dont l'expression la plus authentique serait, selon Adorno, justement le roman de Flaubert). Quelle en serait alors l'issue? "Pour ma part, je la trouve dans mon travail sur Flaubert, qu'on peut d'ailleurs considérer comme un roman. Je souhaite même que les gens disent que c'est un vrai roman" (Sit., IX, p. 123). Quelque temps plus tard, cette idée de "vrai roman" pour désigner L'idiot de la famille est relativisée: " D'abord il faut en venir à l'idée de roman. J'ai peut-être exagéré un peu quand j'ai dit que c'était un roman [...]. Ce roman n'est pas en fait un roman" (Essais sur Sartre, p. 148). Dans ce "vrai roman", dans ce roman qui est et qui n'est pas un roman, réside, selon Sartre lui-même, l'essentiel de son oeuvre: "Le Saint-Genet et L'idiot de la famille me paraissement tout à fait représenter ce que j'ai cherché" (Essais sur Sartre, p. 380). Partant de "l'étude d'un cas concret" (L'Idiot de la famille, I, dorénavant I.F., p. 7) pour tenter de reconstituer l'universalité de toute une époque historique, ce genre de travail biographique (dont l'aboutissement est l'étude sur Flaubert) représente pour Sartre une manière de démontrer sa "méthode progressive-régressive": "L'Idiot de la famille est la suite de Questions de méthode" (I.F., I, p. 7).

Or, il se peut que l'idée conductrice de cette méthode sartrienne d'investigation - l'idée de synthèse universel singulier - ait été esquissée (quoique d'une manière encore très imprécise) au cours de la réflexion faite par Sartre, à la veille de la guerre, sur le roman social américain. Plus que cela, il nous semble que l'Insurrection de 1944 ait eu pour Sartre la valeur d'une preuve - une preuve historique - pour cette idée (envisagée vaguement dans le roman américain) de synthèse entre le singulier et l'universel. Tout se passe comme si la "fulguration" de l'"Apocalypse de 1944", pour utiliser l'expression de Sartre, avait réalisé effectivement un idéal de synthèse que l'auteur, jusqu'alors, poursuivait à tâtons. Mais peut-être le problème n'est-il pas tout à fait bien posé. Il faudrait considérer aussi le sens inverse: cette synthèse que Sartre a vue historiquement réalisée en 1944 se trouverait déjà obliquement anticipée (comme idéal philosophique et littéraire) dans sa réflexion de la veille (ou même du début) de la guerre. Confirmée par la preuve de l'histoire, l'idée sartrienne de synthèse universel-singulier peut se développer jusqu'à prendre la forme achevée d'une méthode qui vise, par le moyen de la biographie historique concrète, à "découvrir en chaque conjoncture, indissolublement liées, la singularité de l'universel et l'universalisation du singulier"' (Sit., IX, p. 190).

La biographie historique concrète, le "vrai roman", comme succédané pour la "Philosophie Traditionnelle" et pour le "Roman Traditionnel"? Sartre ne le dit nulle part, certes. Mais partout dans son oeuvre on peut surprendre, en filigrane, l'idée de biographie comme une sorte d'ersatz des formes philosophique, littéraire et historigraphique traditionnelles - une tentative pour dépasser les impasses de ces formes. En ce sens, on pourrait peut-être considérer la méthode sartrienne d'investigation, le "vrai roman", comme la recherche d'une nouvelle forme de narration (s'il est possible d'adapter cette catégorie à une situation contemporaine) philosophique, littéraire et historique qui présuppose, sous-jacente, une théorie du 
roman indissolublement liée à une théorie de la révolution, plus précisément, à une théorie de la temporalité révolutionnaire qui recèle en elle-même la marque décisive de l'expérience politique de 1944. C'est cela que nous tenterons de développer.

"Flaubert, comme tous ses contemporains, a rendez-vous avec la Révolution de février. [...] Pourtant, il ne fut pas au rendez-vous" (I.F., III, p. 443 et 444). "Il a manqué le rendez-vous de 1848"' (ibid., p. 660). Si Sartre lui-même, comme tous ses contemporains, a rendez-vous avec I'Insurrection de 1944, il n'a pas manqué ce rendez-vous. A la différence de Flaubert, Sartre fut au rendez-vous de 1944 - bien sûr, non pas avec des armes, non pas comme combattant ('je n'ai fait que porter quelques valises', Sit., IX, p. 101), mais comme un écrivain qui veut faire le récit de ce qu'il voit.

"Flaubert est à l'opposé de ce que je suis", affirme Sartre (Interview, Le Monde, le 18 avril 1964). Cela vaut du moins pour le cas de l'attitude des deux écrivains ${ }^{2}$ au moment d'une insurrection révolutionnaire (et aussi pour leur style de raconter ce moment-là, mais j'y reviendrai). La réponse de Flaubert à la "pression de l'Histoire" dit Sartre, c'est "la fuite la plus éperdue": "Jamais il ne s'est senti plus éloigné de la politique: entre février et mai 1848, il a tout autres soucis"' (I.F., III, p. 445 et 446). Ce n'est pas du tout le cas de Sartre. Au contraire de Flaubert - et même de Baudelaire qui "a choisi de vivre le temps à rebours", de "nager à contre-courant" du "grand fleuve" d'idées révolutionnaires du XIX siècle (Sartre, Baudelaire, p. 152-153) -, Sartre se laisse tout à fait emporter par "la force des choses". Pris par ce processus total de transformation historique - processus qui engage la colletivité tout entière -, Sartre, face à "une ville en fusion", découvre à chaud l' "Apocalypse", c'est-à-dire ce moment totalisant et totalisateur capable de supprimer la distance entre l'individu e l'histoire, le singulier et l'universel - moment de "vraie dialectique historique", lit-on dans les Cahiers pour une morale (p. 429). Durant "le triomphe de l'Apocalypse" de 1944 (expression avec laquelle l'auteur désigne cette Insurrection-là, conçue encore comme un instant où l'histoire et son sens se fondent), Sartre voit donc un exemple historique privilégié de synthèse entre le singulier et l'universel.

Comment faire le récit de ce moment de synthèse historique? "Je ne raconte que ce j'ai vu", c'est ainsi que Sartre commence sa série de reportages sur la Libération de Paris. Mais de quelle manière Sartre raconte-t-il ce qu'il voit? Prétendant saisir sur le vif le processus social en cours - un processus de transformation historique accélérée -, Sartre est amené à adopter la rapidité d'un style de reportage socio-journalistique. Nous disions tout à l'heure qu'il va au rendez-vous de 1944 en tant qu'écrivain qui veut témoigner de ce qui se passe. Il faudrait préciser: écrivain-reporter. Comme Maxime du Camp - qui, contre les conseils de son ami Flaubert, se met au milieu de la foule de 1848 ("il veut faire [...] un reportage sur le massacre", I.F., III, p. 446) -, Sartre, "au milieu de la foule de 1944", veut lui aussi

2. Ce qui compte ici, bien entendu, c'est l'interprétation sartrienne de Flaubert. 
faire un reportage, mais non pas du massacre, non pas d'une "révolution manquée", tout simplement d'une Insurrection victorieuse.

Or, cette forme - celle du reportage - adoptée par Sartre pour reconstituer ce moment historique-là de synthèse entre le singulier et l'universel, c'est exactement la forme qu'il avait exaltée, à la veille de la guerre, dans le roman social américain dans ce roman où Sartre semble avoir trouvé la première inspiration pour son idée postérieure d'universel singulier. Loin du style de narration par laquelle Flaubert évoque, dans L'Education sentimentale (que Sartre a d'ailleurs toujours critiqué), la Révolution de 1848, le récit sartrien de 1944 a comme modèle la technique d'un roman né de l'autre côté de l'Atlantique. Il convient donc de regarder en arrière pour essayer de comprendre le sens de ce chemin parcouru par l'auteur.

En 1938, Sartre clôt un article sur Dos Passos avec ces mots apologétiques: "Je tiens Dos Passos pour le plus grand écrivain de notre temps" (Sit., I, p. 24). On risquerait de ne pas bien comprendre la vraie portée d'un si grand éloge, et à quel point il est daté, si l'on ne tenait pas compte de ce que 1938 signifie pour Sartre. La menace imminente de la guerre, tout en "révélant" l'Histoire, révélait en même temps le manque d'instruments théoriques pour la saisir: "L'Histoire [...] m'entourait et m'enserrait comme tous nos contemporains, elle me faisait sentir sa présence. J'étais mal outillé encore pour la comprendre et la saisir, mais pourtant je le voulais fort" (Les camets de la drôle de guerre, dorénavant C.D.G., p. 227). Où trouver des instruments pour la saisir? Pas dans l'"idéalisme" de la tradition culturelle française: "les circonstances nous imposaient de rompre avec nos prédécesseurs: ils avaient opté pour l'idéalisme..."'(Sit., II, p. 256). Le processus de transformation historique radical, "imposant" la nécessité d'une pensée "qui ne fût pas seulement une contemplation" (C.D.G., p. 227) et, par conséquent, ébranlant l'univers de l' "idéalisme" universitaire français, amène Sartre à chercher dans le roman social américain une alternative pour comprendre l' "histoire réelle".

Mais pourquoi la solution pour les problèmes théoriques imposés par les "circonstances" d'une Europe à la veille de la guerre pourrait-elle se trouver dans un roman qui venait d'un autre continent?

A partir de 1938, c'est-à-dire à partir du moment où toute une génération d'intellectuels français perd les illusions d'une "histoire individuelle bien cloisonnée", l'individu, comme l'indique Sartre, ne peut plus être saisi séparément - "il se surprend en voie de généralisation et de dissolution" dans le tout social (Prière d'insérer pour L'Age de Raison et le Sursis, reproduit dans Les Ecrits de Sartre, p. 113). A ce moment-là, Sartre envisage dans le roman social américan un chemin qui permette de développer le problème - mis à l'ordre du jour par le mouvement de l'histoire lui-même - des rapports entre le particulier et l'universel.

Dans l'éloge sartrien de 1938 au roman américain (particulièrement celui de Dos Passos), opposé au roman français, ${ }^{3}$ s'esquisse pour la première fois chez Sartre, à

3. Sartre attribue fréquemment au roman américan la fonction que le "subjectivisme" d'un Proust ou le "naturalisme" 
notre avis, le point de vue qui sous-tendra plus tard son idée d'universel singulier. Regardons-y de plus près: "Comme il est simple, ce procédé, comme il est efficace: il suffit de raconter une vie avec la technique du journalisme américain, et la vie cristallise en social [...]. Du même coup le problème du passage au typique - pierre d'achoppement du roman social - est résolu. Plus n'est besoin de nous présenter un ouvrier-type, de composer, comme Nizan dans Antoine Bloyé, une existence qui soit la moyenne exacte de millers d'existences. Dos Passos, au contraire, peut donner tous ses soins à rendre la singularité d'une vie. Chacun de ses personnages est unique; ce qui lui arrive ne saurait arriver qu'à lui. Qu'importe, puisque le social l'a marqué plus profondément que ne peut faire aucune circonstance particulière, puisque le social c'est lui"' (Sit., I, p. 22). Bien que tout à fait unique, singulier, irréductible, chaque personnage de Dos Passos recèlerait en lui-même le social, ou plutôt c'est le social. Or, ce passage incarne parfaitement l'esprit de l'idée sartrienne d'universel singulier: le singulier portant en soi l'universel, ou inversement, l'universel saisi au coeur même du singulier. Cette idée (qui guidera la "méthode progressive régressive", et particulièrement l'étude sur Flaubert) semble donc avoir connu sa première ébauche chez Sartre au cours de son analyse du roman américain - ce roman qui, tout en montrant le social enclavé dans "la singularité d'une vie", illumine une conjoncture historique vécue par Sartre comme un moment de vraie suppression de la distance entre l'individu et le tout social.

C'est à ce moment-là où la base de la culture française s'effondrait tout en annonçant l'ébranlement de son sol historique - "il nous parut que le sol allait manquer sous nos pas" (Sit., II, p. 242) -, que Sartre, contre la tradition "idéaliste" locale, paraît envisager dans la forme littéraire américaine une piste pour penser un nouveau "réalisme" - "le réalisme de la temporalité" proposé plus tard dans "Qu'est-ce que la littérature?" (Sit., II, p. 327). Le premier signe de ce "réalisme de la temporalité", Sartre l'entrevoit, nous semble-t-il, déjà en 1938 chez Dos Passos: "Le temps de Dos Passos est sa création propre: ni roman, ni récit. Ou plutôt, si l'on veut, c'est le temps de l'histoire" (Sit., I, p. 16). Cette lecture de Dos Passos annonce déjà, dans une conjoncture où, comme le dit Sartre, "l'historicité reflua sur nous" (Sit., II, p. 243), la "littérature de l'historicité" réclamée par l'auteur presque dix années plus tard, en 1947.

Faisant un bilan de l'influence de la littérature américaine sur toute une génération d'intellectuels français qui a développé une "littérature de situations extrêmes" ("Que font Camus, Malraux, Koestler, Rousset etc., sinon une littérature

d'un Zola ("l'objectivité absolue [...] est rigoureusement équivalente à l'absolue subjectivité", Sit., II, p. 328) ne porrait pas accomplir: rendre compte du social dans sa totalité. Si le "subjectivisme" de Proust reste prisonnier de la "vie intérieure" - d'ailleurs son "analyse intellectualiste" " "ne peut opérer (...) que sur un fond d'irrationalité totale" ( L'être et le néant, p. 209) -, le naturalisme de Zola ne nous donne pas non plus le tout social (malgré son propos de le décrire): "Pour les naturalistes, la réalité c'est l'apparence, telle que la science positiviste l'a organisée" (Sit., I, p. 277). Il convient de signaler rapidament que la critique adressée par Sartre au naturalisme est parfois très proche de celle que Lukács, dans son célèbre essai de 1936, "Raconter ou décrire?", lui a aussi adressée. 
dé situations extrêmes?" - Sit., II, p. 327), Sartre écrit: "Quant aux Américains [...], nous avons reconnu en eux des hommes débordés, perdus dans un continent trop grand comme nous l'étions dans l'histoire et qui tentaient, sans traditions, avec les moyens du bord, de rendre leur stupeur et leur délaissement au milieu d'événements incompréhensibles. Le succès de Faulkner, d'Hemingway, de Dos Passos [...] ce fut le réflexe de défense d'une littérature qui, se sentant menacée parce que ses techniques et ses mythes n'allaient plus lui permettre de faire face à la situation historique, se greffa des méthodes étrangères pour pouvoir remplir sa fonction dans des conjectures nouvelles'" (Sit., II, p. 255-256). ${ }^{4}$ Dans cette littérature d'une Amérique sans traditions, lieu d'hommes "débordés", "perdus" au milieu "d'événements incompréhensibles", la "génération intellectuelle" de Sartre, cette génération dont la tradition culturelle venait d'être balayée par l' "ouragan" de l'histoire, perdue elle aussi dans un monde considéré incompréhensible, se reconnaît, c'est-à-dire y voit son propre portrait. Ne pouvant plus se voir reflétée dans le miroir de sa propre histoire culturelle - dans ce miroir qui pendant la "bonace trompeuse", selon les mots de Sartre, avait reflété lumineusement, et "mensongèrement", l'image du beau et "calme jardin de la pensée française", pour utiliser l'expression ironique de Merleau-Ponty -, cette génération cherche son image dans une Amérique elle aussi à la recherche de son identité - terre de "The Misfits", pour me servir du titre d'un film de John Huston. Ce recours aux "méthodes étrangères", méthodes de la littérature d'un monde déchiré, dévoile la situation historique d'une Europe qui semblait aller à la dérive et, plus que cela, anticipe la conjoncture particulière d'une France qui sera bientôt occupée par des étrangers, lacérée, déracinée, dépouillée de ses traditions - devenue "un monde sans tradition", comme l'écrira Sartre dans son journal de guerre ("La mort dans l'âme", Fragments de journal, Oeuvres romanesques, Pléiade).

Empruntant donc, à la veille de la guerre, la technique d'une littérature qui a fleuri ailleurs, en "se greffant des méthodes étrangères", Sartre essaie de se mettre à l'heure du processus social qui se déroule auprès de lui. Cet "emprunt délibéré", pour

4. Encore sur l'influence de la littérature américaine: "Si L'Etranger porte des traces si visibles de la technique américaine, c'est qu'il s'agit d'un emprunt délibéré", affirme Sartre au sujet de l'oeuvre de Camus (Sit., I, p. 106). Et au sujet de sa propre oeuvre littèraire, particulièrement Le Sursis, Sartre, soulignant qu'il a voulu "éviter" la manière dont Zola parle "d'une nation comme d'une seule personne", précise: "J'ai dû avoir recours au "grand écran" [...]. J'ai tenté de tirer profit des recherches techniques qu'ont faites certains romanciers de la simultanéité tels Dos Passos et Virginia Wolf"' (Prière d'insérer pour L'Âge de Raison et Le Sursis, Les écrits de Sartre, p. 113). Cette technique de la simultanéité, ce "recours au grand écran", que Sartre emprunte à la littérature américaine vient surtout du "vrai" cinéma tel que l'auteur le considère - celui capable de peindre "une grande fresque sociale" ("Un film pour l'après-guerre", Les Lettres Françaises, avril 1944). Le cinéma a toujours joué un rôle très important dans la pensée sartrienne. "Il y avait un mode d'expression - écrit Simone de Beauvoir - que Sartre plaçait presque aussi haut que la littérature: le cinéma” (La force de l'âge, p. 59). En ce sens, il convient de rappeler ce discours prononcé par le jeune Sartre: "Je prétends que le cinéma [...] doit servir à votre culture au même titre que le grec ou la philosophie. [...] C'est, de tous les arts, le plus proche du monde réel" ("L'Art cinématographique", reproduit dans Les écrits de Sartre). Ce discours de 1931, exaltant encore la "polyphonie cinématographique" (capacité de développer avec beaucoup de rapiditè "plusieurs thèmes simultanément"), nous aide à mieux comprendre l'idée, présentée par l'auteur plus tard (dans Les Mots, par exemple), de "toucher à l'absolu' à travers le cinéma. 
reprendre l'expression de l'auteur, c'est bien, comme il le dit, "le réflexe de défense d'une littérature" qui se sentait "menacée". Mais c'est en même temps, pour adapter ce qu'Adorno écrit, dans un autre contexte, au sujet de Kafka, "la réponse anticipée à une constitution du monde, où l'attitude contemplative est devenue un sarcasme sanglant, parce que la menace permanente de la catastrophe ne permet plus à personne d'être un spectateur neutre" (Notes su la littérature, p. 24). ${ }^{5}$

Cette "réponse anticipée" à une catastrophe qui s'annonce imminente pourrait être décelée dans un autre article de Sartre, écrit quelques mois avant la déflagration de la guerre, sur le roman américan: "D'où vient que Faulkner et tant d'autres aient choisi cette absurdité-là [...]? Je crois qu'il faut en chercher la raison dans les conditions sociales de notre vie présente. Le désespoir de Faulkner me paraît antérieur à sa métaphysique: pour lui, comme pour nous tous, l'avenir est barré. Tout ce que nous voyons, tout ce que nous vivons, nous incite à dire: "ça ne peut plus durer" et cependant le changement n'est même pas concevable sauf sous la forme de cataclysme. Nous vivons au temps des révolutions impossibles, et Faulkner emploie son art extraordinaire à décrire ce monde qui meurt de vieillesse et de notre étouffement" (A propos de Le Bruit et la Fureur - La temporalité chez Faulkner, juin-juillet 1939, Sit., I, p. 74). Ces mots qui viennent clore l'article pourraient être lus comme une préfiguration du "cataclysme" historique qui éclatera bientôt. C'est sans doute la guerre comme "expérience de l'absurdité du monde" qui se trouve obliquement anticipée dans ce passage sur le roman de Faulkner. Mieux: c'est Sartre lui-même qui décrit la nécessité historique de "ce monde qui meurt de vieillesse" et, ce faisant, parle avant le temps des transformations sociales profondes de l'époque. Cette "réponse anticipée" (qui semble anticiper aussi la réflexion philosophique sur la temporalité dévelopée dans L'être et le néant) présuppose une idée de temporalité qui recèle en soi, comme une plaie sanglante, la marque douloureuse du "désespoir" et de l'"absurdité" (attribués au roman de Faulkner) d'une époque dominée par la terreur du totalitarisme nazi (à la montée duquel Sartre avait personnellement assisté durant son séjor à Berlin, en 1933-1934). C'est à ce moment-là, où "l'avenir est barré", que commence, à partir d'un roman de Faulkner, la réflexion sartrienne sur la temporalité. ${ }^{6}$

Mais qu'est-ce que le temps dans cet essai de 1939? "Le présent de Faulkner est catastrophique par essence: c'est l'événement qui vient sur nous comme un voleur énorme, impensable, - qui vient sur nous et disparaît. Par-delà ce présent il n'y a rien, puisque l'avenir n'est pas'" (Sit., I, p. 66). ${ }^{7}$ Si l'on considère que plus tard Sartre décrira la conjoncture vécue à la veille de la guerre avec le même ton, et les mêmes mots

\footnotetext{
5. "Le survol qu'aimaient tant pratiquer nos prédécesseurs était devenu impossible", affirme Sartre en 1947 (Sit., II, p. 242-243).

6. "Et voici qu'à présent j'entrevois une théorie du temps", écrit Sartre, quelques mois après, dans Les carnets de la drôle de guerre, p. 257.

7. "Le Futur n'a pas d'être en tant que Futur", lit-on dans L'être et le néant (p. 168).
} 
("les événements fondaient sur nous comme des voleurs", Sit., II, p. 254), qu'il décrit la temporalité dans le roman de Faulkner, on peut mieux comprendre tout le sens de sa profonde "admiration" pour cet écrivain: le présent "catastrophique" de Faulkner dit au sujet du présent historique de la France de 1939 ce que Sartre alors ne parvenait pas, ou ne pouvait pas encore, dire complètement. La temporalité tragique de ce roman américain illumine le temps historique d'une France qui marchait vers une tragédie. Regardons-y mieux.

En 1947, réflechissant sur cette conjoncture de 1939 ("où la pression historique nous écrasait" - Sit., II, p. 236), Sartre décrit ce qu'il pressentait alors: "quelque chose nous attendait dans l'ombre future, quelque chose qui nous révélerait à nous-mêmes peut-être [...] avant de nous anéantir; le secret de nos gestes [...] résidait en avant de nous dans la catastrophe à laquelle nos noms seraient attachés'" (Sit., II, p. 243). Face à impossibilité de freiner cette marche tragique vers la "catastrophe" (présentée comme une sorte de néantisation), face à l'impossibilité d'ordonner, à la manière de Faust, "Temps, suspends ton vol", la réflexion sartrienne de 1939 sur le roman de Faulkner, exprimant obscurément ce qui semblait alors un inévitable travail de destruction fait par Saturne, transforme le temps en destin. A ce moment-là où "les jeux sont faits" ("nous n'avons rien à faire: nous n'avons plus jamais rien à faire", écrit Sartre, peu avant la capitulation, dans son journal de guerre, p. 1561), le processus historique en cours apparaît obliquement à l'intérieur de la réflexion sartrienne comme l'incarnation de la fatalité propre à une grande tragédie. "Tout est absurde", affirme Sartre reprenant ensuite ces mots de Macbeth: "La vie est une histoire contée par un idiot, pleine de bruit et de fureur, qui ne signifie rien" (Sit., I, p. 73).

"La plupart des grands auteurs contemporains [...] ont tenté de mutiler le temps [...] Proust et Faulkner l'ont simplement décapité, ils lui ont ôté son avenir"' (Sit., I, p. 71). Mais dans cette analyse sartrienne de 1939, c'est le temps qui, "comme un voleur énorme, impensable", mutile et décapite l'homme, déchire son être, dévore son avenir: "L'homme passe sa vie à lutter contre le temps et le temps ronge l'homme comme un acide, l'arrache à lui-même et l'empêche de réaliser l'humain"' (Sit., I, p. 37). ${ }^{8}$ Dans cette image d'un temps qui déchire et dévore - image qui pourrait bien évoquer le Saturne peint par un Rubens ou par un Goya -, il est possible d'entrevoir le portrait d'uneépoque "pleine de bruit et de fureur" d'une vraie tragédie. Ou, plus particulièrement, le portrait (préfiguré) d'une France qui marche vers le fatidique 13 juin 1940: "Paris est déclaré ville ouverte", on lisait ce jour-là partout sur les affiches. Le lendemain la Wehrmacht pénétrait dans un "Paris désert" - une "ville sans regard" (Die Stadt ohne Blick), diront les Allemands. Sans regard et sans avenir - "l'occupation a dépouillé les hommes de leur avenir", écrit Sartre (Sit., III, p. 29) -, cette France qui venait d'entrer à

8. Dans son journal de guerre, Sartre, utilisant la même image de déchirement de l'essai de 1939, écrit: "La guerre [...] ronge patiemment les oeuvres de l'homme et parfois, entre les choses à demi digérées, [...] un rapport neuf et maléfique s'établit brusquement"' (La mort dans l'âme, p. 1571) 
l'heure allemande ${ }^{9}$ est le symbole d'une temporalité tragique qui dévore tout un monde et condamne une génération à "persévérer sans espoir" (Sit., II, p. 254).

C'est la fatalité de la capitulation de la France et, comme on le croyait alors, l'irréversibilité de la marche de l'Allemagne nazie vers une hégémonie européenne ("temps irréversible", dit Sartre), qui se trouverait ainsi obliquement préfigurée dans la réflexion sartrienne faite a la veille de la guerre. Tout en voulant parler de la temporalité dans un roman américan, Sartre parle également (quoique de manière voilée) de la temporalité historique de la France. Plus que cela, on pourrait peut-être dire que l'essai de 1939 sur Faulkner anticipe le statu quo de la France de 1940, ou mieux, ce serait une sorte de contemporain philosophico-littéraire du présent politique de la France de la débâcle. C'est cette France déchirée de 1940, où l'on attend la mort "comme une victime propitiatoire" (selon Sartre dans son journal de guerre, p. 1570), qui s'insinue déjà dans la réflexion de 1939 sur la temporalité dans le roman de Faulkner, cette temporalité "catastrophique" qui conduit fatalement à la mort.

Traversée par le mouvement de l'époque, la réflexion sartrienne de la veille de la guerre - cette sorte de chronique oblique d'événements "catastrophiques" pourrait être considérée comme une anticipation du "monologue intérieur de la France occupée" mentionné dans Qu'est-ce que la littérature? (Sit., II, p. 258). L'idée d'une temporalité qui réalise un travail de destruction "irréversible", contre lequel toute résistance serait inutile, nous donne l'image préalable du processus que l'on appelle souvent "dégradation de l'esprit public" pendant la "drôle de guerre" - processus qui débouche sur le "fatalisme" et sur la "résignation" de 1940: "un monde qui ne résiste jamais"; "on ne nous avait demandé que notre patience", écrit Sartre dans son journal de guerre (p. 1575 et 1578). ${ }^{10}$

Ne pouvant plus faire l'histoire ("nous pouvons souhaiter la victoire des Anglais ou des Allemands mais nous nous sommes mis hors de jeu, totalement neutralisés", lit-on dans La mort dans l'âme, p. 1584), les héritiers de l'effrondrement de 1940 la conçoivent comme une sorte de "course à l'abîme" où, pour me servir de la manière dont le jeune Sartre avait défini la tragédie, "tous les personnages, quoi qu'ils disent, quoi qu'ils fassent, avancent vers leur fin" ("L'art cinématographique", Les Ecrits de Sartre, p. 549). Cette idée d'une temporalité tragique (préfigurée, comme nous venons de le voir, dans l'essai sur Faulkner) amène Sartre à penser le processus historique en cours comme un mécanisme de forces aveugles, irrationnelles, absurdes, comparables aux phénomènes de la nature. D'où le recours aux métaphores de grandes

9. Et cela même au sens littéral car, comme on le sait, les horloges furent alors avancées d'une heure.

10. A propos de l'état d'esprit dominant à l'époque, c'est intéressant de rappeler ce passage de Julien Gracq, tiré de son Balcon en forêt: "Pour la dernière fois peut-être en 1914 les hommes étaient partis avec l'idée de rentrer pour les vendanges: en 1939 [...] ils savaient au fond d'eux-mêmes qu'ils ne reverraient qu'une terre où serait passé le feu". Après l'armistice, comme l'indiquent les documents de l'époque, "la conviction que l'Europe serait allemande" est devenue un sentiment presque hégémonique parmi les contemporains. " $\mathrm{C}$ 'est le coeur serré que je vous dis qu'il faut cesser le combat" - ordonna Pétain le 17 juin. "Chacun est rentré chez soi" , c'est ainsi que Jean Cassou évoque l'état de "résignation" régnant en 1940. "Il y a un vide énorme. (...) Nous avons été pris d'un immense dégoût pour cette guerre ratée", écrit Sartre, en août de 1940, dans son journal (La mort dans l'âme, p. 1583). 
catastrophes naturelles pour désigner la conjoncture de 1939-1940: "nous étions au centre d'un cyclone"; "le déluge est venu" (Sit., II, p. 252 et 259). "Cyclone", "déluge", ou encore "catastrophe céleste" et "cataclysme" - nous voilà parmi des tragédies de la nature que nul ne pourrait éviter. "Contraints par les circonstances à découvrir la pression de l'histoire, comme Torricelli a fait de la pression atmosphérique..." (Sit., II, p. 251). Or, cette identification récurrente entre processus historique et processus naturel exprime parfaitement la réalité d'une France dépouillée de son propre temps historique: "Nous vivons sans mémoire et sans avenir, dans l'instant", écrit Sartre aprés la défaite (La mort dans l'âme, p. 1583). "Sans mémoire et sans avenir", cette France de 1940, condamnée à durer "dans l'instant", à durer en deçà du temps (car l'instant, selon L'être et le néant, p. 170, est "intemporel"), est conçue comme étant en dehors du processus historique - jetée hors de l'histoire: "Nous sommes [...] stérilisés: des vivants éternels, comme des protozoaires", lit-on encore dans le journal de Sartre (p. 1582). "Hors de jeu", "neutralisée", "anéantie", la France de ces "années terribles", cette France dont la mémoire historique fut arrachée par un "voleur impensable", privée ainsi de ses "grands souvenirs" (expression par laquelle Marx désignait le "culte du passé" révolutionnaire français), plongea dans la durée d'une "nuit du non-savoir" (Sit., II, p. 251) - domaine de forces obscures et incompréhensibles. "Tout notre univers s'était effondré, nous ne comprenions rien", dit une contemporaine. ${ }^{11}$

Que reste-t-il dans cette France qui, comme l'affirme Sartre, "a perdu ses souvenirs"? "La mort dans l'âme", si l'on pense au titre du journal de guerre de l'écrivain (titre repris, comme on le sait, pour le troisième tome des Chemins de la liberté). On pourrait ajouter, pour utilizer une expression de L'être et le néant, un énorme "pouvoir néantisant". "Il n'y a que la Mort qui se voit", écrit Sartre en juin 1940. "Mort: guerre morte, mort dans le ciel, ville morte [...] et nos coeurs que nous avons tués cet hiver, par crainte de souffrir" (La mort dans l'âme, p. 1565). "Le grand escamotage historique" de ces années (Sit., II, p. 242) condamne toute une génération à durer dans un monde déraciné: "Comme les champignons [...] nous vivons à fleur de terre. Et lorsqu'un camp meurt [...] il pourrit et sèche à la surface du sol et se transforme en poussière anonyme sous le soleil"' (La mort dans l'âme, p. 1581).

"Goût amer" d'une époque qui pourrait bien incarner concrètement cette définition sartrienne du Néant: "Le néant est la possibilité propre de l'être et son unique possibilité" (L'être et le néant, dorénavant E.N., p. 117). Au moins à ce moment-là, moment de "mort dans l'âme" et dans les choses, l'idée abstraite d'un Néant qui "hante l'être" (ce Néant qui est "la condition première [...] de toute enquête philosophique ou scientifique", E.N., p. 46) ne deviendrait-elle pas historiquement concrète? Serait-ce exagéré de dire que nulle part, hormis cette France "anéantie" par "la dureté des temps"' (Sit., II, p. 251), on ne pourrait voir, dans une pareille pureté,

11. Témoignage extrait du documentaire "Femmes dans la guerre", une série de cinq émissions présentées par G. Guidez. 
"le néant iriser le monde, chatoyer sur les choses", bref, "éclore dans le monde"? (E.N., p. 58). Si dans cette conjoncture-là le néant était en fait l' "unique possibilité" de l'être, il y aurait peut-être lieu de penser que l'image abstraite du monde présentée dans "ce couple indissoluble, L'être et le néant" (E.N., p. 159) expose obliquement la réalite d'une France dont l'être historique fut anéanti - la France de la fatalité du "calvaire" de l'occupation, comme l'écrit Sartre dans Les Lettres Françaises (clandestines).

Et pourtant c'est justement dans L'être et le néant que l'on voit s'esquisser, pour la première fois chez Sartre, l'idée d'une temporalité qui guérit - idée qui rompt avec le caractère de fatalité (présent, comme nous l'avons vu, dans l'essai de 1939) du travail de destruction effectué par le temps: "Le temps ronge et creuse, il sépare, il fuit. Et c'est encore à titre de séparateur - en séparant l'homme de sa peine ou de l'objet de sa peine - qu'il guérit" (E.N., p. 169). Ici, encore comme dans l'essai sur Faulkner, le temps "ronge et creuse", mais son travail de destruction n'est plus fatal. Le même mouvement qui mème à la destruction peut mener aussi à la guérison. Le temps n'est don plus pensé comme destin tragique; son mouvement n'apparaît plus comme irréversible ou univoque.

Ce dépassement de l'idée d' une temporalité "catastrophique" (dont le travail de destruction est irréversible, c'est-à-dire auquel il est impossible de résister) dans l'idée d'une temporalité qui guérit, qui est salut, pourrait être considéré comme l'autre aspect - l'aspect philosophique - de l'itinéraire historique de la France: de la "catastrophe" de 1939-1940 à la Résistance. Bien que ne se mouvant qu'à l'intérieur de la problemátique traditionnelle de la philosophie, la réflexion sur la temporalité développée dans L'être et le néant semble détecter obscurément, à l'insu de l'auteur, la réalité du mouvement de Résistance qui alors se faisait déjà sentir.

Il est désormais devenu possible, comme l'idée sartrienne d'une temporalité qui guérit le laisse entrevoir, de résister à l' "irréversibilité" d'un temps qui, en 1939, mutilait les hommes en leur "volant" l'avenir et en écrasant tout espoir. Le cours de l'histoire va bientôt défaire le mythe de l'irréversibilité de la victoire nazi dans l'Europe. Le mouvement de résistance, dans la France et en dehors de la France, commence à ronger ce mythe qui, jusqu'alors, avait été vécu comme destin inévitable. D'abord, les Anglais tinrent le coup. On sait, par les mémoires d'epoque, à quel point la résistance anglaise représenta pour les contemporains un ébranlement de la réputation d'invincibilité de la Wehmacht. ${ }^{12}$ En France, même si la Résistance ne fut durant longtemps qu'une "anarchie combattante", selon l'expression de de Gaulle, elle permit à toute une génération de ne plus vivre l'histoire comme destin, ou plutôt de "briser le destin", pour utiliser une expression de Sartre. Si les jeux ne sont pas faits, il devient possible d'échapper à la durée d'un "monde qui ne résiste jamais".

12. "Si nous pouvons tenir tête, toute l'Europe recouvrera un jour sa liberté", disait Churchill en juin 1940. 
"Résister: c'est le cri de vous tous qui ne vous résignez pas [...]. Mais c'est surtout agir, [...] reprendre le combat" - proclame le numéro 1 de Résistence (bulletin officiel du Comité national de salut public, 15 décembre 1940, l'une des premières feuilles clandestines). Si la "catastrophe" de 1940 a pu signifier le "vol" des "grands souvenirs" du passé révolutionnaire français, la Résistance représente une tentative délibérée de récupération de ce passé. ${ }^{13}$ "La Patrie en danger - 1792-1943: comme nos grands aïeux les volontaires de la levée en masse - Parisiens aux armes!", lit-on, par exemple, sur une affiche des Francs-tireus et partisans. Tout au long de ce passage de la France de l'"Effondrement" à la France du "Choix", pour me servir des deux sous-titres du film de Marcel Ophuls sur l'époque, "Le chagrin et la Pitié", un vocabulaire nouveau s'impose: action, choix, liberté. Si dans la France de la débâcle on était condamné à ne rien faire, dans la France de la Résistance ${ }^{14}$ on est condamné au choix, c'est-à-dire "condamné à être libre" (E.N., p. 168), pour mettre le problème dans les termes de L'être et le néant (puisque choix et liberté sont identifiés dans ce livre).

La Résistance met ainsi à l'ordre du jour le problème (que Sartre avait déjà envisagé dans la philosophie de l'histoire d'Aron) du libre choix dans l'histoire: "Pendant la Résistance, en effet, il semblait y avoir une possibilité de décision libre", dit Sartre (Sit., IX, p. 100). Et dans La République du Silence, l'auteur affirme que, durant la Résistance, "le choix que chacun faisait de lui-même était authentique puisqu'il se faisait en présence de la mort" (Sit., III, p. 12). ${ }^{15} \mathrm{C}$ 'est donc la question du "choix historique", dévelopée philosophiquement dans L'être et le néant, que la Résistance incarne concrètement.

D'après L'être et le néant, comme on le sait, c'est par la signification donnée au passé (problème de la révision du passé à partir d'une décision, ou du sens d'une décision, prise dans le présent) qu'une société humaine "devient historique", "s'historialise" (E.N., p. 557). Dans cette perspective, on pourrait dire que la "décision" de la Résistance de redonner un sens au passé révolutionnaire français (reprendre ce passé "à titre de monument", pour utiliser le langage de L'être et le

13. D'où, par exemple, le titre d'un éditorial du Combat en 1944: La France perdue et retrouvée. Cette France "perdue et retrouvée", selon le journal, c'est la France des révolutions.

14. Ce qui compte ici, bien entendu, ce n'est pas la réalité de la Collaboration, mais plutôt comment toute une génération, et particulièrement Sartre, a élaboré le "mythe" de la Résistance. "Les forces de la Résistance sont presque un mythe", écrit Sartre dans ses reportages de 1944.

15. Dans ce sens, rappelons encore ce passage de Simone de Beauvoir: "Quand [...] les Français ont dû décider de leur attitude en face de l'occupant [...], ils ont dû choisir librement [...]" (L'Existentialisme et la Sagresse des Nations, Nagel, p. 93-94). Faisant référence à la France de l'occupation, Merleau-Ponty écrit: "Nous avons vécu [...] un de ces moments où l'histoire en suspens, les institutions menacées exigent de l'homme des décisions fondamentales [...]" (Humanisme et Terreur, p.43). Si Sartre a converti, comme le dit T. Adorno, la catégorie kierkegaardienne de décision en "croyance dans la liberté absolue de décision" (Dialéctica Negativa, Madrid, Taurus, p.55), c'est parce qu'il généralise théoriquement l'expérience politique de la Résistance. D'ailleurs, Sartre lui-même, critiquant ses premières pièces, affirme: "pendant la Résistance [...] il fallait accepter les risques de l'action [...]. J'en ai conclu que, dans toute circonstance, il y avait toujours un choix possible. C'était faux"' (Sit., IX, p.100) 
néant), signifierait pour Sartre le retour de la France au temps historique. Tout se passe comme si "la dynamique temporelle" (qui "correspond à l'écoulement matériel ou, suivant la terminologie kantienne, au cours du temps", E.N., p. 169) allait finalement prévaloir sur "ce que Kant appelle l'ordre du temps" (qui "se définit tout d'abord par l'irréversibilité", E.N., p. 169).

"Tout maintenant est destiné à devenir un autrefois", lit-on dans L'être et le néant (p. 169). Espoir face à une temporalité qui se constitue comme telle parce que, en tant que changement, elle dépasse la durée, la répétition, le permanent, l'identique: "Sans changement point de temporalité, puisque le temps ne saurait avoir prise sur le permanent et l'identique" (E.N., p. 182). Espoir face à une temporalité qui permette donc de dépasser l' "absurde" d'une situation décrite par Sartre de la manière suivante: "La présence de la mort au bout de notre route a dissipé notre avenir en fumée, notre vie est "sans lendemain", c'est une succession de présents" (Sit. I, p. 108).

On ne pourrait pas oublier, bien sûr, que L'être et le néant ne se propose que de décrire des essences philosophiques - c'est d'ailleurs, comme l'indique son propre titre, un "essai d'ontologie phénoménologique". Mais ces "essences" philosophiques semblant dévoiler, d'une certaine façon, l'"essence historique" d'une France qui devait faire face à des "situations extrêmes", pour reprendre l'expression de Sartre. S'il se peut que, comme nous l'avons suggéré, l'image abstraite du monde, présentée dans ce livre, exprime (obliquement et à l'insu de l'auteur, il convient d'insister là-dessus) ${ }^{16}$ un processus de “néantisation" réel, il se peut également qu'elle exprime aussi, à travers la réflexion sur la temporalité, la réalité, ou plutôt le "mythe", de la Résistance. Mieux: c'est le passage d'une France qui demeurait inchangée dans la durée de l'instant, en deçà du temps, dominée par des forces aveugles et irrationnelles, à une France qui retrouve son temps historique, qui "s' historialise" par l'action libre de la Résistance (car "la liberté étant choix est changement", E.N., p. 553), qui semble être annoncé dans la réflexion sur la temporalité développée dans L'être et le néant. Conçu abstraitement "pendant l'hiver 1939-1940", 17 écrit entre l'automme 1941 et le début de 1943, ce livre anticiperait l'été "apocalyptique" d'août 1944 (j'y reviendrai plus tard).

"Il fallait nous sauver ou nous perdre [...]". C'est ainsi que l'auteur évoque, dans Qu'est-ce que la littérature?, cette conjoncture de la guerre (Sit., II, p. 253). Le salut, comme le dit Sartre au sujet de Proust, serait "dans le temps même" (Sit., I, p. 70). Ou, plus précisément, le salut viendrait incarné historiquement dans le mouvement de la Résistance et philosophiquement dans l'idée d'une temporalité qui guérit. De l'écrasement de l'espoir à l' "invention" d'un "espoir neuf" (pour utiliser les mots de

16. Faisant un bilan de l'influence de la Guerre sur sa pensée, Sartre dit: "En fait, j'aurais dû commencer à découvrir cette force des choses dès L'être et le néant" (Sit., IX, p. 99). Mais ensuite il ajoute: "L'être et le néant retrace une expérience intérieure sans aucun rapport avec l'expérience extérieure - devenue, à un certain moment, historiquement catastrophique - de l'intellectuel petit-bougeois que j'étais" (p. 102).

17. "Pendant l'hiver 1939-1940, j'étais déjà en possession de la méthode et des conclusions principales" - affirme Sartre, dans Questions de Méthode (p. 42), au sujet de L'être et le néant. 
Sartre à propos du Traître, d'André Gorz - ce "premier livre d'après la défaite", Sit., IV , p. 81): le même temps qui détruit peut guérir, peut mener à la Libération.

Cette idée de salut par le temps présuppose à la fois "un souvenir et une anticipation", pour reprendre, dans un autre contexte, une piste indiquée par Sartre lui-même au cours de son analyse sur la Révolution Française (Questions de méthode, p. 94). Ce serait un souvenir du passé révolutionnaire de la France et une anticipation de l'itinéraire de la Résistance vers la Libération. C'est ce temps perdu durant l'occupation et reconquis par "l'Apocalypse de 1944" - le temps de la Révolution qui semble être préfiguré dans l'idée d'une temporalité qui guérit.

L'Insurrection de 1944 - ce moment de "joie devant l'Apocalypse" - amène cette idée sartrienne d'une temporalité qui guérit à prendre la forme précise de l'apologie de la violence révolutionnaire comme salut. "Le moment de l'Apocalypse", lit-on dans les Cahiers pour une morale (écrit à la suite de L'être et le néant, comme on le sait), c'est à la fois "le moment de la fête", de la "générosité", et "le moment de la violence" (p. 429-430). On verra l'aboutissement de cette idée dans la célèbre préface de 1961, Les Damnés de la Terre, au livre de Franz Fanon: "Guérirons-nous? Oui. La violence, comme la lance d'Achille, peut cicatriser les blesures qu'elle a faites"' (Sit., V, p. 192). Cette violence qui, au lieu de détruire, guérit, Sartre a cru la voir réalisée en août 1944.

Ce chemin qui mène de l'idée d'une temporalité tragique et destructice à l'idée de salut à travers l'action régénératrice de la temporalité révolutionnaire, c'est-à-dire de la violence révolutionnaire, passe donc nécessairement par l'Insurrection de 1944 - ce moment de "passage d'un monde ossifié et refroidi à une Apocalypse", pour reprendre la manière dont Sartre décrit l'instant d'explosion révolutionnaire dans la Critique de la Raison Dialectique (dorénavant C.R.D., p. 484). ${ }^{18}$ A L'origine de l'apologie sartrienne de la violence révolutionnaire se trouve sans doute cette "semaine d'Apocalypse" qui guérit les blessures faites par la guerre et par l'occupation: "Tout Paris a senti, dans cette semaine d'août, que les chances de l'homme étaient encore intactes, [...] ces quelques jours ont suffi pour prouver la puissance de la liberté" ( $\mathrm{La}$ Libération de Panis: une semaine d'Apocalypse, in Les Ecritis de Sartre). L'espoir mort en 1940, renait avec le printemps de la Résistance et devient célébration de l'Apocalypse sous la "haute température" politique des barricades d'août 1944.

"Il ne peut y avoir de liberté que dans la libération" - écrit Sartre, généralisant philosophiquement l'expérience politique de 1944, dans les Cahiers pour une morale (p. 430). Et pour qu'il y ait libération on ne pourrait pas se passer de violence: "comme la liberté opprimée veut se libérer par la force, l'attitude révolutionnaire exige une théorie de la violence comme réplique à l'oppression" - lit-on dans "Matérialisme et Révolution" (Sit., III, p. 217). Dans la C.R.D., cette idée prend une forme plus définie:

18. J'ai essayé de montrer ailleurs ("Le thème de la Révolution dans la pensée de Sartre", Études Sartriennes, IV, Cahiers de Sémiotique textuelle, $n^{\circledR} 18$, Publidix, 1990) que le concept sartrien de "groupe en fusion" a été peut-être forgé à chaud pendant les événements de la "semaine d'Apocalypse" de 1944. Sartre lui-même a assisté à la formation d'un groupe en fusion et a tenté de décrire ses structures dans sa série de reportages pour Combat. 
"La terreur, c'est la violence de la liberté commune contre la nécessité" (p. 529). D'où l'explication de la Terreur en tant que "contre-violence" (C.R.D., p. 537). C'est encore cette idée de violence révolutionnaire comme antidote contre la violence elle-même qui sous-tendra les analyses de Sartre sur mai 1968: "Cette violence des étudiants [...] n'est, en réalité, qu'une contre-violence. [...] Ils font office de contrapoison. [...] La violence des étudiants et des jeunes travailleurs n'a jamais été que défensive" (Sit., VIII, p. 175, 176 et 179).

En essayant de comprendre ce qu'íl appelle, dans ses reportages de 1944, "naissance de l'esprit insurrectionnel", Sartre arrive ainsi à une théorie de la temporalité révolutionnaire. Comme réponse théorique au défi politique vécu, l'auteur érige la Révolution en principe conducteur de sa pensée: "La vraie culture, c'est la Révolution; cela veut dire qu'elle se forge à chaud" (Sit., V, p. 172). Et dans Qu'est-ce que la littérature?, on lit: "Le règne des fins ne se peut réaliser sans Révolution" (Sit., II, p. 298).

Mais quelle forme historique de révolution Sartre a-t-il en vue? "Nous devons dans nos écrits militer en faveur de la liberté de la personne et de la révolution socialiste" (Sit., II, p. 298). Cela impliquerait, comme prétend l'auteur, un dépassement de l'"étroitesse" du mot "révolution" lorsqu'il est identifié uniquement à une révolution bourgeoise, c'est-à-dire lorsqu'on désigne par "révolution" une insurrection politique et qu'on néglige "l'aspect économique" de ce changement social. Révolution, dit Sartre, c'est "un phénomène historique comportant à la fois le changement du régime de la propriété, le changement du personnel politique et le recours à l'insurrection" (Sit., II, p. 302). "C'est surtout un processus total de transformation historique qui, selon l'interprétation faite dans la C.R.D., II de "la théorie de la révolution permanente" (qui viendrait plus de Marx que de Trotsky), "doit, d'un même mouvement dialectique, s'approfondir sans cesse en dépassant ses propres objectifs (radicalisation) et s'étendre de proche en proche à tout l'univers (universalisation)" (C.R.D., II, p. 110-111). "Processus de totalitarisation", apocalyptique, qui, comme l'affirme l'auteur dans Questions de Méthode, engendre "l'universel concret" - idée dont le germe se trouverait déjà chez Robespierre: "l'universel chez lui est concret (il est abstrait chez les autres constituants) et [..] il se confond avec l'idée de totalité. La révolution [...] est une totalité en devenir qui doit se réaliser un jour comme totalité devenue"' (p. 106).

Or, cet "universel concret" qui "se confond avec l'idée de totalité" - ébauché dans la pensée de Robespierre, dans cette "dialectique naissante" -, Sartre le voit incarné dans le Résistant. Porte-parole de la collectivité, le Résistant est celui qui "se choisissant lui-même dans sa liberté, choisissait la liberté de tous'" (Sit., III, p. 14). Il n'est donc pas conçu comme un simple individu, c'est plutôt le singulier qui porte en lui l'universel. Ce que Sartre dit au sujet de l'homme en général - "un homme n'est jamais un individu; il vaudrait mieux l'appeler un universel singulier" (I.F., I, p.7) exprime parfaitement son point de vue sur le Résistant, et en particulier sur le rôle qu'il a joué pendant l'“Apocalypse de 1944". 
"Dans l'Apocalypse - lit-on dans la C.R.D. - [...] l'unité synthétique est toujours ici; ou, si l'on préfère, en chaque lieu de la ville, à chaque moment, dans chaque processus partiel, la partie se joue tout entière et le mouvement de la ville y trouve son achèvement et sa signification" (p. 461). Dans cette perspective, on pourrait dire, pour reprendre notre fil conducteur, que "l'unité synthétique" réalisée en 1944 signifierait pour Sartre non seulement la réconciliation avec l'esprit de 1789 mais aussi la preuve historique de l'idéal de synthèse - entrevu, à la veille de la guerre, dans le roman américain - entre le singulier et l'universel. Désormais, Sartre n'a plus besoin de chercher cette synthèse ailleurs: 1944 fournit, sous ses yeux, ce qu'il poursuivait dans le roman de l'autre côté de l'Atlantique. Célébrée comme un instant de vraie communion entre le singulier et l'universel, de synthèse historique, la "semaine d'Apocalypse" c'est le moment où la pensée de Sartre peut rejoindre la tradition révolutionnaire française. C'est le moment où toute une "génération intellectuelle" peut de nouveau se sentir chez elle, et, pour me servir librement des mots avec lesquels Hegel exalte l'avènement de la conscience de soi, peut enfin, "tel le marin après un long périple sur une mer déchaînée, crier: Terre". Il suffit maintenant de franchir la Seine pour retrouver, dans "toute une ville aux barricades", son propre sol historique, son passé révolutionnaire. "Dans le présent le passé restauré", comme disait Baudelaire.

Outre la réalisation effective de l'idéal de synthèse envisagé dans le roman américain, l'expérience révolutionnaire de 1944 semble aussi avoir représenté pour Sartre la réalisation de l'idéal de la "phílosophie concrète" que, contre l' "idéalisme" universitaire français, il cherchait, à l'avant-guerre, dans la phénoménologie allemande. Que l'on se souvienne de ses mots dans l'essai de 1939 sur Husserl: "La philosophie française, qui nous a formés, ne connaît plus guère que l'épistémologie. Mais pour Husserl et les phénoménologues, la conscience que nous prenons des choses ne se limite point à leur connaissance. [...] Husserl a réinstallé l'horreur et le charme dans les choses. [...] Nous voilà délivrés de Proust. Délivrés en même temps de la "vie intérieure" [...]. Ce n'est pas dans je ne sais quelle retraite que nous nous découvrirons: c'est sur la route, dans la ville, au milieu de la foule, chøse parmi les choses, hommes parmi les hommes" (Sit., I, p. 31-32). C'est cette "philosophie concrète" que Sartre tentait de trouver à partir de Husserl (une équivoque colossale, comme diraient par exemple Horkheimer et Habermas), ${ }^{19}$ cette philosophie amalgamée aux situations concrètes du monde, que l'auteur voit confirmée "au milieu de la foule" de 1944.

Ce qu'il y a de commun entre les essays sur le roman américan et sur la phénoménologie allemande, c'est justement la quête d'une pensée concrète qui puisse "rajeunir" la culture française, pour utiliser le terme employé par Sartre dans Les camets de la drôle de guerre (p. 228). La quête d'une pensée capable de saisir le "coeur

19. Comme on le sait, Horkhemeir et, après lui, habermas ont critiqué Husserl justement en tant que representant de la "Théorie Traditionelle", cette pensée qui succombe à l' 'ilusion de la théorie pure". 
même des choses", comme le dit l'auteur dans son éloge de Faulkner (Sit., I, p. 67). C'est la "découverte" de la "force des choses" qui s'anticipe déjà dans ces essais de la veille de la guerre.

"Je reviens à chercher une solution réaliste", déclare Sartre, durant la "drôle de guerre", tout en annonçant qu'il avait enfin découvert que la philosophie de Husserl "évoluait vers l'idéalisme" (C.D.G., p. 227). C'est cette "solution réaliste" que l'auteur entrevoit maintenant, d'une manière paradoxale, dans la philosophie de Heidegger: "Suffisamment détaché de Husserl, désirant une philosophie pathétique, j'étais mûr pour comprendre Heidegger. [...] Heidegger est l'apparition dans le monde d'une conscience libre [...]. Et son époque c'était précisément une époque tragique d"Untergang" et de désespoir pour l'Allemagne. [...] L'attitude de Heidegger est évidemment un dépassement libre vers la philosophie de ce profil pathétique de l'histoire. [...] Ainsi puis-je retrouver cette assomption de son destin d'Allemand dans l'Allemagne misérable d'après-guerre pour m'aider à assumer mon destin de Français dans la France de 1940" (C.D.G., p. 227-229). Cette "philosophie pathétique" d'une "époque tragique" pour l'Allemagne pouvait, certes, exprimer d'une certaine façon le "profil pathétique de l'Histoire" dans la France de 1940. Mais plus que cela, l'assimilation sartrienne de cette "apparition dans le monde d'une conscience libre", de cette philosophie "héroïque" (telle que Sartre définit encore la philosophie heideggerienne), semble déjà préfigurer le "programme libre et héroïque" de la Résistence. On pourrait synthétiser en disant que ce "programme" fait dans la réalité ce que Sartre envisageait à la fois dans la forme fictionnelle du roman (américain) et dans la forme idéale de la philosophie (de Husserl d'abord et de Heidegger après).

“Gide [..] et moi [...] nous n'étions que trop disposés à prendre le réel pour un décor", écrit Sartre dans Les carnets de la drôle de guerre après avoir souligné qu'ils partageaient tous les deux un certain "manque" du "sens du réel" - "les événements les plus importants" leur semblaient des "mascarades" (C.D.G., p. 391-392). Simple manque du "sens du réel"? Dans le cas de Sartre, en effet, on pourrait dire qu'il pense "les évéments les plus importants", par exemple une explosion révolutionnaire, et très particulièrement celle d'août 1944, justement comme des moments où la réalité prend la forme d'un décor. D'où l'aspect fictionnel du récit sartrien de l'expérience politique de 1944. Dans une forme quasi romanesque, Sartre raconte l'Insurrection comme une histoire dont les personnages sont des héros combattants, c'est-à-dire les personnages historiques sont recréés presque comme des personnages de fiction. "Carnaval de guerre", "fête tragique et mortelle", "moment d'ivresse et de joie" - voilà quelques expressions avec lesquelles l'auteur, dans sa série de reportages ainsi que dans l'article La Libération de Paris: une semaine d'Apocalypse, reconstitue la "cérémonie solennelle" de 1944. Plutôt qu'un simple manque du "sens du réel", ce que l'on voit s'esquisser dans le récit sartrien de 1944 est une certaine conception 
du réel (plus précisament, du moment révolutionnaire) comme décor, scène, spectacle - réalité historique et fiction amalgamées. ${ }^{20}$

$\mathrm{Au}$ fond ce que j'ai toujours désiré passionnément, [...] c'est d'être au centre d'un événement beau. Un événement [...] qui ne soit pas en face de moi comme un tableau on un air de musique, mais qui soit fait autour de ma vie et dans ma vie, avec mon temps. Un événement [...] dont je sois l'auteur, comme le peintre est l'auteur de son tableau. Ce désir [...] de la beauté temporelle..." - écrit Sartre en février 1940 (C.D.G., p. 343). Cet "événement beau" viendrait effectivement sous la forme de l'Apocalypse de 1944 - "le plus beau souvenir de notre vie", comme l'évoque Simone de Beauvoir. ${ }^{21}$ Vécue comme un moment de "beauté temporelle" (de là une certaine esthétisation sartrienne de la Révolution), cette "semaine d'Apocalypse" incarne parfaitement l'événement tant idéalisé "fait autour de ma vie et dans ma vie, avec mon temps". Recréée dans une forme quasi fictionnelle, l'expérience de 1944 devient une sorte de personnage principal du roman philosophique sartrien: un événement "dont je sois l'auteur, comme le peintre est l'auteur de son tableau".

F. Jameson a très bien montré (dans Marxism and Form, Princeton University Press, 1971) que l'analyse sartrienne sur la Révolution française cherche à "recréer synthétiquement dans la pensée", à la manière d'un romancier historique, l'expérience historique concrète. Si dans "la vie des coutumes et des traditions - écrit Jameson rien effectivement ne change ni ne se passe, il n'y a rien pour conter dans le sens narratif, [...] la particularité du moment révolutionnaire c'est qu'en lui l'histoire prend la forme d'événements narrables, elle se révèle avec une continuité, avec un début, un milieu et une fin". On pourrait ajouter (bien que Jameson ne mentionne pas le rôle de l'Insurrection de 1944 dans la pensée sartrienne) que la "semaine d'Apocalypse" - moment où la vie quotidienne acquiert une grande valeur, où il y a quelque chose "pour conter dans le sens narratif", où "l'histoire prend la forme d'evénements narrables", ou même la forme d'un décor - amène Sartre à développer le problème (déjà esquissé par Roquentin dans La Nausée) de la recréation de l'action historique et, du même coup, ouvre le chemin à la "méthode progressive-régressive".

Quelques mois avant la Libération de Paris, Sartre, réfléchissant sur l'oeuvre de Brice Parain, parle de "réaliser l'universel avec sa propre chair" ("comme dans le système de Kant") et de "découvrir soudain l'universel au coeur de l'individuel" (à partir de la dialectique hégélienne entre l'individuel et l'universel) (Sit., I, p. 208-209). L'Insurrection de 1944, conçue exactement comme un moment où chaque individu réalise "l'universel avec sa propre chair", a dans la pensée sartrienne le pouvoir de consolider les bases d'une méthode calquée sur l'idée de synthèse entre le singulier et l'universel. Légitimée par la preuve de l'histoire, cette idée peut se développer jusqu'à son aboutissement dans l'étude sur Flaubert - cette monumentale tentative

20. En ce sens, que l'on se souvienne que Sartre, critiquant Jules Renard, dit qu'il lui manque "la puissance nécessaire pour reconstruire la réalité [...] comme Proust" (C.D.G., p. 422).

21. Film "Simone de Beauvoir", série Témoins, de J. Dayan et M. Ribovska, 1983. 
pour reconstituer l'universalité d'une époque historique à partir de la singularité d'une oeuvre. Il nous semble ainsi possible de dire que la "méthode progressive-régressive" - cette méthode qui vise la "vraie dialectique du subjectif et de l'objectif" (Questions de méthode, p.80) - aurait cristallisé théoriquement l'"unité synthétique" de 1944. Sartre aurait élevé l'experiénce historique vécue à la condition de méthode d'investigation philosophico-littéraire. En réalisant historiquement la synthèse que l'auteur poursuivait dans la forme fictionnelle du roman et dans la forme idéale de la philosophie, l'Apocalypse de 1944 finit par définir la physionomie elle-même de la pensée sartrienne: philosophie et littérature (forme fictionnelle)22 liées de l'intérieur par une théorie de la révolution. Mieux: ni philosophie "pure" ni littérature "pure", mais plutôt un mouvement de passage entre les deux fait par la médiation d'une théorie de la révolution. "Suis-je philosophe? Ou suis-je littéraire? Je pense que ce que j'ai apporté depuis mes premières oeuvres, c'est une réalité qui soit les deux: tout ce que j'ai écrit est à fois philosophie et littérature, non pas juxtaposées, mais chaque élément donné est á fois littéraire et philosophique" (entretien avec M. Sicard, Essais sur Sartre, p. 380). Une théorie de la temporalité révolutionnaire soustend cette liaison intime de philosophie et de littérature, pourrait-on ajouter. La "soudaine découverte", en 1944, de "l'universel au coeur de l'individuel" fonde ainsi une théorie de la temporalité révolutionnaire qui recèle en elle-même, indissolublement liés, roman, philosophie et révolution.

Si notre lecture est exacte, il devient possible d'adapter à Sartre lui-même ce qu'il a dit au sujet de Flaubert: "Plus tard, après réflexion, il mettra en place un schème historique qui lui permettra de comprendre les événements de 1871 et rétrospectivement ceux de 1848. [...] Et l'Education sentimentele [...] sortira de cette longue rumination d'un événement qu'il n'a point vécu" (I.F., III, p. 448). Mais c'est Sartre lui-même qui, après la Libération, se met à la recherche d'un "schème" philosophique et historique (d'où sa "rencontre" avec le marxisme) qui lui permette de vraiment comprendre août 1944 et rétrospectivement 1917, 1871, 1848, 1830, 1789. Bref, un "schème" pour rendre compte du problème de la Révolution. Dans ce sens, on pourrait dire que la Critique de la raison dialectique et L'idiot de la famille sortiront "de cette longue rumination d'un événement" que Sartre, contrairement à Flaubert, a vécu intensément. II pensera désormais l'histoire dans la perspective de la Révolution, ou mieux, la Révolution comme "le moment décisif de l'histoire" (Sit., X, p. 219).

Ce n'est donc pas par hasard que le problème de la révolution se trouve au fond de L'idiot de la famille. C'est un livre d'histoire: la révolution de 1848, etc." - voilà comment Sartre définit son "étude sur Flaubert" (Interview, Tribune Étudiante, n 56, janvier-février, 1962). Ce qui est en jeu dans cette étude c'est, comme le dit l'auteur dans un entretien de 1976, "le sens de la réalité de 1848" ("Essais sur Sartre", p. 148). Le propos de Sartre est de reconstituer, à travers la singularité d'une oeuvre, le

22. "J'utilise la fiction guidée, contrôlée, mais fiction quand même", affirme Sartre au sujet de son étude sur Flaubert (Sit., IX, p. 123) 
processus social qui aboutit au "massacre de 1848" (Sit., IX, p. 119). Cependant, le fait d'avoir en vue le problème de la Révolution n'implique pas, bien sûr, que la particularité de Flaubert soit négligée. Il s'agit plutôt, d'après ce qu'on lit déjà dans Questions de méthode, de comprendre Flaubert "comme universalité vécue dans la particularité" (p. 110). Ce que Sartre veut faire dans le cas de Flaubert c'est bien ce qu'il avait exalté, comme nous l'avons vu, dans la méthode de Dos Passos: "rendre la singularité d'une vie" tout en montrant qu'elle recèle en elle-même le social. L'idiot de la famille représente justement une tentative pour saisir l'oeuvre de Flaubert comme un moment unique et irréductible de l'histoire du roman qui porte, dans sa singularité, le sens de toute une époque - "c'est le social", comme disait Sartre au sujet des personnages de Dos Passos.

Mais, plus encore que le roman social américain, c'est le référentiel marxiste ${ }^{23}$ (outre la psychanalyse) qui est sollicité par Sartre dans L'idiot de la famille pour accomplir son projet biographique annoncé dès L'être et le néant: "L'unification irréductible que nous devons rencontrer, qui est Flaubert et que nous demandons aux biographes de nous révéler [...] " (E.N., p. 620). Et quelques pages après: "Cette psychanalyse n'a pas encore trouvé son Freud; tout au plus peut-on en trouver le pressentiment dans certaines biographies particulièrement réussies. Nous espérons pouvoir tenter d'en donner ailleurs deux exemples, à propos de Flaubert et de Dostoievski" (E.N., p. 635). Pour aller plus loin, c'est-à-dire pour que ce projet biographique abstraitement envisagé devienne concret sous la forme proposée dans le tome III de L'idiot de la famille - étudier Flaubert et "l'Esprit objectif au début de la seconde moitié du XIX siècle" -, Sartre est amené à chercher "la totalisation par des méthodes marxistes" (Sit., X, p. 103). Plus précisement, L'idiot de la famille veut de propos délibéré "remettre sur ses pieds" l' "idéalisme" de la notion hégélienne d'"Esprit objectif" et "indiquer la fonction instrumentale" que cette notion "peut remplir dans la perspective du matérialisme historique" (I.F., III, p. 43). Dans la "perspective", donc, du matérialisme historique, Sartre prétend, à travers l'étude sur Flaubert, mettre à l'épreuve son idée de dialectique (développée dans la C.R.D.) comme universel singulier. Partir d'un cas particulier pour atteindre "l'objectivité de l'histoire" - voilà le grand projet sartrien.

Ce projet d'une monographie historique concrète traverse l'oeuvre sartrienne tout entière. Déjà dans L'Imaginaire, Sartre indique, d'une manière encore vague, que l'on pourrait récupérer, à partir de la singularité du David de Michel-Ange, la vérité historique de la Renaissance. Mais c'est sans aucun doute dans Les camets de la drôle de guerre que l'on voit s'esquisser pour la première fois chez Sartre, tout au long de son analyse des rapports entre l'empereur Guillaume II et l'avènement de la Première Guerre, l'idée de monographie historique comme alternative pour les impasses de l'historiographie traditionnelle: "C'est la méthode historique et les préjugés

23. "J'essaie de donner une méthode plus ou moins révolutionnaire parce qu'elle est marxiste", affirme Sartre à propos de L'Idiot de la Famille (Sit., X, p. 114). 
psychologiques qui la gouvernent - et non la structure même des choses - qui produisement cete division des facteurs de l'histoire en couches signifiantes parallèles. Ce parallélisme disparaît si on traite le personnage historique à partir de l'unitè de son historialisation. Mais je reconnais que ce que je crois avoir montré n'est valable que dans le cas où l'étude historique est une monographie... " (C.D.G., p. 386-387). Sartre ajoute que la biographie historique pourrait être un "correctif synthétique de la décomposition abstraite" opérée par la méthode histonique traditionnelle (laquelle sépare ce qui, en réalité, n'est que "l'unité d'un même mouvement" - C.D.G., p. 401-402).

C'est dans cette perspective - la "perspective de la biographie", comme on lit dans Questions de méthode - que la "méthode progressive-régressive" va essayer de reconstituer la totalisation du processus historique. "Il ne suffit pas - affirme Sartre utilisant l'exemple de Robespierre - de le montrer poussé par le mouvement de la Révolution; il faut savoir aussi comment il s'inscrit en elle. Ou, si l'on veut, de quelle Révolution il est l'abrégé, la vivante condensation. Et c'est cette dialectique qui seule permettra de comprendre Thermidor" (Questions de méthode, p. 107). Pour échapper à la "fausse universalité" (dont "Marx était si loin") d'un certain "marxisme paresseux" (qui "dissout le particulier dans l'universel" "Hegel, du moins, laissait subsister le particulier en tant que particularité dépassée"), "la méthode euristique doit envisager le "différentiel" (le terme est emprunté à Merleau-Ponty) - voilà le point de vue dèveloppé dans Questions de méthode (voir en particulier les pages $48,49,107$ ). ${ }^{24}$

Il n'est donc pas fortuit, comme Jameson l'a bien remarqué (dans Marxism and Form), que le premier héros sartrien ait été un biographe - Roquentin. D'ailleurs, déjà dans le prière d'insérer de la première édition de La Nausée (reproduit dans Les Ecrits de Sartre) Sartre présente le travail du biographe comme un travail d'historien. En effet, nous semble-t-il, Roquentin anticipe certains problèmes théoriques que la guerre, et la "révélation" de l'Histoire qui en résulte, posera pour Sartre. Tout se passe comme si, à partir des Carnets de la drôle de guerre, Sartre lui-même tentait de donner continuité, à la lumière de l'expérience historique vécue, au projet biographique de Roquentin et à ses interrogations sur le problème de la reprise du passé par le présent. De l'aventurier imaginaire du XVIII siècle, M. de Rollebon, étudiè par Roquentin, Sartre passe, dans un premier moment, au personnage historique réel Guillaume II. On connait la suite: la tentative pour comprendre Brice Parain comme "un homme de l'entre-deux-guerres" (dans Aller et Retour), l'étude sur Baudelaire, Jean Genet, Mallarmé, Tintoret, Joseph LeBon (manuscrit inédit), le scénario Freud (pour le film de John Huston), Staline (particulièrement dans la

24. La critique développée par Sartre, particulièrement dans Questions de Méthode, contre le marxisme mécaniste (qui n'engendre qu'un "squelette d'universalité", comme le dit encore l'auteur) reprend le point de vue soutenu par Merleau-Ponty dans les années 40. “Être marxiste [...] ce n'est pas renoncer à l'individu [.... . C'est bien rejoindre l'universel, mais sans quitter ce que nous sommes" - écrit Merleau-Ponty dans "La Guerre a eu lieu" (Sens et Non-Sens, Nagel, p. 264-265). Ou encore: "Nous n'atteignons pas l'universel en quittant notre particularité, mais en faisant d'elle un moyen d'atteindre les autres" ("Le Métaphysique dans l'Homme", Sens et Non-Sens, p. 162). 
C.R.D., II). ${ }^{25}$ Si L'idiot de la famille représente l'aboutissement de ce long itinéraire historico-biographique, il ne faudrait pas oublier le grand projet sartrien (irréalisé) de faire avec Robespierre (et la Révolution de 1789) ce qu'il a fait avec Flaubert (et la Révolution de 1848). Et il ne faudrait pas oublier non plus que cette entreprise biographique sartrienne est aussi une entreprise autobiographique: "Une autobiographie, mais plutôt sociale et politique qu'individuelle, sur l'évolution des gens de ma génération, de 1905 à la Libération, en passant par 1914, 1930, 1940. A travers mon histoire, c'est celle de mon époque que je veux transcrire" (interview, Libération, 4 novembre 1953).

D'après Jameson (Marxism et Form), c'est la pratique historiographique de Michelet qui marque le passage d'une histoire écrite à partir du point de vue de la "vie individuelle" à une "histoire de la collectivité", ou mieux, "le passage de l'individualisme (avec son culte implicite de la personnalité) à un nouveau type de narration collective" (où le héros c'est le "peuple", et non plus un certain personnage individuel, comme le dit Michelet). Que l'on se souvienne en outre de la manière dont Michelet lui-même présente "la Méthode et l'Esprit" de son Histoire de la Révolution française: "Que d'hommes en un homme! [...] Rembrandt a fait trente portraits de lui, je crois, tous ressemblants, tous différents. J'ai suivi cette méthode. Si l'on prend la peine de suivre dans ces deux volumes chacun des grands acteurs historiques, on verra que chacun d'eux a toute une galerie d'esquisses, touchées chacune à sa date [...]. Nous nous sommes dit et répété un mot qui nous est resté présent et qui domine ce livre: l'histoire, c'est le temps" (Pléiade, v. 1, p. 290-291). Et dans sa conclusion, Michelet écrit: "Ou'est-ce que l'histoire? La spécification. Plus elle spécifie, précise, caractérise, plus elle est historique, plus elle est elle-même. Mon mérite, celui de ce livre, c'est son constant effort pour échapper aux vagues généralités, pour retrouver la personnalité, la pénétrer en soi, la suivre en ses variations, la noter jour par jour" (v. 2, p. 995). Portrait biographique et temporalité historique (plus précisément, temporalité révolutionnaire) - voilà le binôme qui pourrait très bien résumer le propos sartrien de chercher dans chaque "cas particulier" la synthèse des déterminations historiques de son époque. On atteint l'histoire à travers la "spécification", dit Michelet. A travers le "différentiel", dit Sartre, grand lecteur de Michelet. Trouver l'universel au coeur du singulier ce serait la manière d'"échapper aux vagues généralités", pour reprendre Michelet, ou d'échapper à la "fausse universalité", pour reprendre Sartre. Plutôt qu'une image cristallisée, le portrait d'un individu doit, aussi bien pour Sartre que pour Michelet, receler en lui-même le mouvement totalisateur du temps historique.

C'est dans cette synthèse entre biographie et histoire que la méthode sartrienne envisage le chemin pour accomplir ce que l'auteur appelle "universalisation lente qui

25. Perry Anderson définit (In the Tracks of Historical Materialism) le projet du tome II de la C.R.D. comme une "tentative pour construire l'intelligibilité de toute une époque historique et d'une formation sociale - l'URSS de 1930 à 1950 - à travers la figure de Staline comme instance finale d'unification". 
se réalise par l'affirmation et le dépassement du particulier" (Sit., IV, p. 80). Amalgamant tout au long de son itinéraire depuis la technique du roman américain jusqu'aux "totalisations marxistes", passant sans doute par la pratique historiographique de Michelet, la méthode sartrienne, sous la forme de la monographie historique concrète, semble vouloir atteindre une issue pour les impasses, non pas seulement de l'historiographie traditionnelle, mais aussi de la philosophie et du roman traditionnels. Nous revenons ainsi à notre point de départ. Le "vrai roman" comme ersatz des formes philosophique et romanesque?

Adorno affirme, dans un autre contexte, que la littérature biographique "est un produit de la décomposition de la forme romanesque" (Notes sur la littérature, p. 38). Et encore: le reportage et le cinéma auraient privé le roman "de bien des tâches qui lui revenaient traditionnellement" (p. 38). Or, c'est exactement dans le reportage, dans "la technique du journalisme américain" - cette technique de la simultanéité, du "recours au grand écran" -, que Sartre, comme nous l'avons vu, entrevoit d'abord la méthode qui puisse conduire à la synthèse universel-singulier. Après avoir lui-même mis cette technique à l'épreuve dans son journal de guerre, ${ }^{26}$ dans les journaux clandestins et surtout dans ses reportages de 1944, Sartre trace le profil des Temps Modemes, lors de son premier numéro, par la voie du reportage: "Il nous paraît, en effect, que le reportage fait partie des genres littéraires et qu'il peut devenir un des plus importants d'entre eux. La capacité de saisir intuitivement et instantanéament les significations, l'habileté à regrouper celles-ci pour offrir au lecteur des ensembles synthétiques immédiatement déchifrables sont les qualités les plus nécessaires au reporter; ce sont celles que nous demandons à tous nos collaborateurs" ("Présentation des Temps Modernes", Sit., II, p. 29-30). Et dans Qu'est-ce que la littérature?, tentant de caractériser ce qu'il appelle "une littérature de la Praxis", Sartre écrit: "Ceux d'entre nous qui ont collaboré aux feuilles clandestines, s'adressaient dans leurs articles à la communauté entière.[...] La littérature de la résistence n'a pas produit grand-chose de bon. Mais cette expérience nous a fait pressentir ce que pourrait être une littérature de l'universel concret" (Sit., II, p. 257). L'expérience de la littérature de résistance offre ainsi à Sartre une piste pour penser un nouveau type de relation entre reportage, forme journalistique à l'américaine, roman, philosophie et révolution. C'est de cette idée d'une "littérature de l'universel concret", née de l'expérience de la Résistance, que viendrait le "réalisme de la temporalité" (préfiguré à la veille de la guerre, comme nous l'avons vu, dans la réflexion sur le roman américain), dont l'aboutissement est le "vrai roman".

"Ce qui fait, je crois, l'originalité de notre position, c'est que la guerre et l'occupation, en nous précipitant dans un monde en fusion, nous ont fait, par force, redécouvrir l'absolu au sein de la relativité même" - écrit Sartre dans son bilan de

26. Au sujet de La mort dans l'âme, fragments de journal, Michel Contat écrit: "On y voit Sartre aux prises avec une forme qu'il avait déjà exploitée, dans La Nausée, celle du journal, qu'il tend à tirer, d'un côté, vers le pur récit romanesque à l'américaine (relation sans commentaires de faits et de comportements) et de l'autre, vers la méditation. Si cette dernière, dans La Nausée, était métaphysique, elle commence ici à devenir à la fois historique et existentielle" (Oeuvres Romanesques, Plélade, p. 2134). 
1947 (Sit., II, p. 245). En essayant de rendre compte de cette "redécouverte" de "l'absolu au sein de la relativité même", Sartre, fondant technique journalistique, roman, philosophie et révolution dans la forme de la biographie historique, envisage un nouveau type de narration philosophico-littéraire. ${ }^{27}$

Ce qui est en cause dans cet itinéraire de la pensée sartrienne - de L'être et le néant au "vrai roman" - c'est, nous semble-t-il, le problème de la forme de survivance de la philosohie et de la littérature dans le monde contemporain. On pourrait peut-être dire que la monographie historique concrète a dans la pensée de Sartre la même fonction que le roman comme forme narrative avait, selon la lecture de Jameson, dans la pensée du jeune Lukács: faire face aux problèmes que la théorie abstraite, la "philosophie pure", ne peut pas résoudre, réaliser la synthèse (qui se donnait en tant que forme idéale dans l'Esprit Absolu hégélien) entre le sujet et l'objet, l' "esprit" et la "matière". "Le roman, comme forme, c'est la tentative, dans les temps modernes, pour récupérer quelque chose de la qualité de la narration épique, en tant que réconciliation entre esprit et matière [...], sous conditions de vie qui dorénavant rendent l'épopée impossible" - c'est ainsi que Jameson, dans Marxism and Form, présente la thèse de la Théorie du Roman de Lukács. Mais dorénavant, d'après Adorno, c'est la narration elle-même, comme forme immanente au "roman traditionnel", qui est devenue impossible dans les conditions sociales de la vie contemporaine. Si, après la "décomposition de l'Esprit Absolu", la "philosophie pure", comme l'indique Lukács dans Histoire et Conscience de Classe, s'est révélée incapable de saisir le tout social (ce n'est pas par hasard que le marxisme commence par la critique de la philosophie et aboutit à la critique de l'économie politique), après la "décomposition de la forme romanesque", selon Adorno, le roman en tant que forme narrative n'est plus, comme le pensait le jeune Lukács de la Théorie du Roman, l'instrument privilégié pour dévoiler la réalité historique.

Bien que formulés d'une façon tout à fait différente par Sartre, ce sont ces problèmes concernant le statut de la philosophie ("il ne peut pas y avoir des philosophes en ce moment", Sartre au Brésil, La Conférence d'Araraquara) et de la littérature ("il n'y en a plus, de littérature", Sit., X, p. 114) à notre époque qui semblent être au fond de la recherche sartrienne d'un nouveau "réalisme" capable de "comprendre le monde social" (Questions de méthode, p. 123). D'où l'idée de monographie historique comme succédané contemporain pour la "Philosophie Traditionnelle" et pour le "Roman Traditionnel". Du Lukács de la Théorie du Roman au Sartre des reportages et des monographies historiques, ce qui est en jeu c'est, pour ainsi dire, l' "idéal du concret", la tentative pour récupérer effectivement, chacun à sa manière et d'après les conditions sociales du moment, "quelque chose de la qualité

27. Jameson parle, dans Marxism and Form, de "praxis biographico-narrative" chez Sartre. Michel Contat, à son tour, écrit: "la philosophie de Sartre (son matérialisme historique subjectif, si l'on veut) est dans sa démarche même une philosophie narrative: le roman et l'autobiographie (le roman autobiographique) sont constitutifs de son expression" (préface aux Oeuvres Romanesques, Pléiade, p. XII). 
de la narration épique", c'est-à-dire la synthèse entre subjectivité et objectivité, particulier et universel, "esprit et matière". Dans les deux cas, pourrait-on ajouter, il s'agit d'un même propos (malgré la différence substantielle entre leurs instruments théoriques) de rendre compte de la totalité historique. ${ }^{28}$ Ou mieux, d'un propos de saisir dans la pensée, à travers une théorie de la révolution (qui n'est pas la même dans les deux cas, bien entendu), le mouvement historique réel: "c'est l'événement qui doit être écrit littérairement et qui, en même temps, doit donner un sens philosophique" - dit Sartre au sujet de sa méthode (Essais sur Sartre, p. 380). Si le "réalisme", comme l'écrit Jameson glosant les thèses du jeune Lukács, "dépend, de même que la praxis révolutionnaire elle-même, de moments historiques privilégiés où l'accès au social en tant que totalité peut à nouveau être réinventé", il nous paraît possible de considérer la "réinvention" sartrienne du "réalisme", sous la forme du "réalisme de la temporalité", comme une tentative contemporaine pour acclimater l'idéal de synthèse de la vraie narration aux nouveaux temps, aux Temps Modemes d'une France profondément marquée par l'expérience de la Guerre, de l'Occupation, de la Résistance et de l'Insurrection de 1944. De même que pour le jeune Marx la Révolution devait réaliser l'idéal de la philosophie (idée reprise par Lukács dans Histoire et Conscience de Classe) et pour le premier Lukács (selon l'interprétation de Jameson) la Révolution devait réaliser l'idéal du roman, pour Sartre, comme nous l'avons vu, c'est la Révolution (incarnée particulièrement dans l' "Apocalypse" de 1944) qui réalise son idéal philosophico-littéraire de synthèse entre l'universel et le singulier. ${ }^{29}$

Il est vrai que, dans un certain sens, la théorie sartrienne de la Révolution tend à généraliser - de là son aspect plus problématique - philosophiquement et littérairement l'expérience politique de 1944. Philosophiquement: l'expérience historique concrète devient souvent à l'intérieur des analyses de Sartre presque une catégorie spéculative - et dans ce cas on ne pourrait pas parler de dépassement mais plutôt de simple reprise de la forme spéculative traditionnelle. Littérairement: travaillant avec le "mythe" de la Résistance, la méthode "fictionnelle" sartrienne tend

28. Lorsque Lukács, en 1947, critique l' "existentialisme français" - critique qui a déclenché, comme on le sait, une polémique avec Sartre et Merleau-Ponty -, il ne le fait pas à partir du point de vue de ses premières oeuvres (lesquelles il avait déjà reniées), mais à partir du point de vue officiel de l'orthodoxie soviétique.

29. Réfléchissant sur le "marxisme occidental", et faisant une analogie entre l'oeuvre de Sartre sur Flaubert et celle de Benjamin sur Baudelaire, Perry Anderson écrit que "l'esthétique est devenue le pont par lequel la philosophie tente de s'approcher du monde concret" (Considerations of Western Marxism). Mais après avoir reconnu cela, Perry Anderson, généralisant, affirme curieusement que le "marxisme occidental" comme un tout aurait renversé l'itinéraire de Marx (de la philosophie vers l'économie politique), c'est-à-dire qu'il serait retourné aux domaines de la "philosophie pure". D'un côté, c'est bien vrai que les représentants du "marxisme occidental" étudiés par Perry Anderson (spécialement Lukács, l'École de Francfort et Sartre) n'ont pas fait la critique de l'économie politique. Mais ce qu'ils ont élaboré est, comme nous essayons de le montrer dans le cas particulier de Sartre, quelque chose de beaucoup plus complexe qu'un retour pur et simple à la "philosophie pure". En óutre, dans le cas spécifique de Sartre il n'y aurait pas de sens de parler de "renversement" de l'itinéraire de Marx parce que l'auteur n'a ni commencé par l'économie politique (au contraire, il a commencé par la "philosophie pure" telle qu'elle se définit dans L'être et le néant) ni n'est jamais passé par elle. 
à traiter les personnages historiques réels comme des personnages de fiction-c'est bien dans ce sens un "vrai roman". Mais, en sens inverse, ce "vrai roman" est en même temps un "roman vrai" : 30 à l'intérieur de ce mouvement spéculatif de la pensée sartrienne il y a une expérience historique réelle; sa méthode fictionnelle reproduit, "retotalise", recrée, ou même anticipe, un processus historique concret. Imprégnée d'un bout à l'autre du mouvement de son époque, l'oeuvre sartrienne est à la fois fiction et vérité historique. De là vient sa fécondité.

De là vient aussi son succès à l'après-guerre, faudrait-il ajouter. "Il y avait, du moins à première vue, un remarquable accord entre ce qu'il apportait au public et ce que celui-ci réclamait" - écrit Simone de Beauvoir à propos du succès de Sartre ( $\mathrm{La}$ Force des Choses, p. 62). Mais qu'est-ce que ce public réclamait? Ou, formulant la question différemment, de quelle façon particulière Sartre exprime-t-il son époque?

"Ces jeunes lecteurs sont défaitistes" - affirme Sartre au sujet du public de Flaubert: "ils demandent à leurs écrivains de montrer que l'action est impossible, pour effacer leur honte d'avoir raté leur Révolution" (Questions de méthode, p. 59). Or, on pourrait dire exactement le contraire d'une France qui venait de fêter sa Libération. Dans cette France de la "fraternité virile", pour utiliser une expression récurrente chez Malraux et chez Camus ("nous avons vécu les années de la fraternité virile", écrit ce dernier dans ses Écrits Politiques, p. 20-21), il n'y a pas de place pour des défaitistes: ce que l'on réclame c'est plutôt une philosophie de l'action, de l'engagement, de la "liberté héroïque" pour "effacer" la "honte" de la défaite de 1940. L'oeuvre sartrienne, incarnant théoriquement le "mythe" de la Résistance, offre à toute une génération la possibilité de se racheter de cette "honte" - antidote, donc, contre le "chagrin" de l' "effondrement". C'est tout le "charme" de la tradition révolutionnaire française, pour me servir de l'expression d'Engels, qui sera désormais solennellement restitué au public sous la forme d'une philosophie de l'"Apocalypse".

"Il viendra un moment où Flaubert paraîtra en avance sur son époque [...] parce qu'il est en retard sur elle, parce que son oeuvre exprime sous un masque à une génération dégoûtée du romantisme les désespoirs post-romantiques d'un collégien de 1830" - lit-on dans Questions de méthode (p. 58). Et dans L'idiot de la famille on retrouve la même idée: "L'auteur ne peut être contemporain de ses contemporains que s'il est, tout ensemble, en retard sur eux et en avance. Bien souvent d'ailleurs pour ne pas dire toujours - l'avance est déterminée par le retard"' (I.F., III, p. 421). Rien de plus vrai dans le cas de Sartre lui-même. Elaborée durant les sombres moments de l'Occupation, la théorie sartrienne d'une temporalité qui guérit est, comme nous avons essayé de le montrer, à la fois une reprise du passé révolutionnaire de la France (il suffit de rappeler la conception apocalyptique, professée par les révolutionnaires du

30. "Je voudrais qu'on lise mon étude comme un roman [...] Je voudrais en même temps qu'on le lise en pensant que c'est la vérité, que c'est un roman vrai" - dit Sartre au sujet de L'Idiot de la famille (Sit., X, p. 94). 
XVIII siècle, de la Révolution comme salut, rédemption) et une anticipation de l'"Apocalypse" de 1944. Mieux: en récupérant le passé révolutionnaire, cette théorie anticipe le présent politique de 1944 - elle devient, avant le temps, contemporaine de ce présent. Le retard (l'attachement aux idées en cours au XVIII siècle) résulte donc en une avance (l'image préalable de la "liberté héroïque" de la Résistance). Comme le dit encore Sartre, "dans nos sociétés en mouvement les retards donnent quelquefois de l'avance" (Les Mots, p.55).

Le lecteur de Sartre à l'après-guerre (ce lecteur qui a vécu la "résignation" de 1940 et les espoirs nés de la Résistance) reconnait dans le mouvement de la pensée sartrienne (de l'idée d'une temporalité qui détruit fatalement à l'idée d'une temporalité qui guérit) le mouvement de sa propre histoire sociale et politique. Du "chagrin" de l' "effondrement" au salut par un temps qui guérit, par une philosophie de la liberté et de l'engagement - voilà ce que l'oeuvre sartrienne offre, à partir de 1945, à un lecteur "dégoûté" de l'expérience de l'Occupation et enivré par la "liberté renaissante", pour employer une expression récurrente dans la presse de l'époque.

"L'époque nous servit" - écrit Sartre dans Merleau-Ponty vivant -: "il y avait entre Français une transparence des coeurs, inoubliable, qui était l'envers de la haine. A travers cette amitié qui préférait tout d'avance en chacun pourvu qu'il détestât les nazis, nous nous reconnûmes; les mots essentiels furent dits: phénoménologie, existence; nous découvrîmes notre vrai souci" (Les Temps Modemes, n 184-185, 1961, p. 307). Mais, faudrait-il insister, ces "mots essentiels" furent dits avant même ce moment de "fulguration de l'existence", tel que Merleau-Ponty le définit dans le numéro inaugural des Temps Modemes ("La guerre a eu lieu"). En ce sens, on pourrait dire au sujet du cas particulier de L'être et le néant ce que Sartre dit à propos de l'oeuvre de Parain et de Proust: "Il souffre d'un retard léger, il n'a pas été transmis à l'heure fixée - tout juste comme l'oeuvre de Proust, écrite avant la guerre de 1914, lue après - et c'est à ce retard, à cette dissonance légère, qu'il devra sa fécondité" (Sit., I, p. 180). Écrit pendant la guerre et lu après, ${ }^{31}$ L'être et le néant souffre lui aussi d'un "retard léger". Et si dans ce cas pareillement la fécondité vient du retard, c'est parce qu'il est la "réponse anticipée", pour reprendre l'expression d'Adorno, à la constitution d'un nouvel ordre social - plus précisément, à la constitution d'un monde qui, tout en sortant de la lutte contre le totalitarisme nazi, érige la liberté comme "mot essentiel". C'est l'image abstraite de ce monde que nous trouvons obliquement préfigurée dans L'être et le néant.

Ayant donc anticipé le vocabulaire d'une époque emportée par la "puissance de la liberté", d'après la définition de Sartre lui-même, la philosophie sartrienne peut trouver à l'après-guerre l'énorme succès qu'elle a connu. Si 1940 "pèse comme un cauchemar",

31. "Achevé a u début de 1943, le livre parut en été et, étant donné les circonstances, passa un peu inaperçu. Il fallut attendre 1945, année où la vogue soudaine de Sartre attira sur son oeuvre maîtresse une attention qu'aucun ouvrage philosophique n'avait connue précédemment, pour que les commentateurs s'en emparent [...]" - lit-on dans Les écrits de Sartre (p. 86). 
pour utiliser l'expression de Marx, sur une France qui, en 1945, tente de retrouver sa place dans le monde, la philosophie sartrienne de la "liberté hérö̈que" permet d'exorciser ce cauchemar et de reconquérir l'espoir. C'est dans cette conjoncture que Les Temps Modernes naissent avec un propos délibéré: "Notre préoccupation doit être de servir la littérature en lui infusant un sang nouveau, tout autant que de servir la collectivité en essayant de lui donner la littérature qui lui convient" ("Présentation de Temps Modernes", Sit., II, p. 30). A ce moment-là où toute neutralité, toute "attitude contemplative", pour revenir aux mots d'Adorno, est devenue un "sarcasme sanglant", l'oeuvre sartrienne, en évoluant vers une pensée de l'engagement, donne effectivement à la collectivité ce qui lui convient. Ici, encore une fois, la réflexion sartrienne sur Flaubert peut illuminer (bien que cette lumière soit presque toujours oblique) l'itinéraire de Sartre lui-même: "Flaubert se voit voler son oeuvre, il ne la reconnait plus [...]. Quelle pouvait donc être cette époque pour qu'elle réclamât ce livre et pour qu'elle y retrouvât mensongèrement sa propre image? "(Questions de méthode, p.113). Dans la pensée sartrienne de l'engagement, dans cette incarnation de l'esprit de la Résistance "heroique", le public reconnaît ("mensongèrement", quand on pense qu'en fait la presque totalité des Français, même si elle n'a pas collaboré, du moins n'a pas résisté) l'image qu'il réclame, c'est-à-dire celle qui lui convient. Inutile de rappeler la perplexité des "existentialistes" face au succès soudain de leur mouvement de rénovation culturelle (et face aussi à cette étiquette qui leur fut attribuée). "Porte-parole des autres", comme le dit Geneviève Idt faisant référence, dans un exposé inédit, justement au succès de l' "écriture sartrienne", Sartre représente pour toute une génération une sorte de metteur en scène dont la caméra, par la technique de la simultanéité, "permet de situer notre histoire dans l'univers entier", pour reprendre les mots apologétiques de l'auteur au sujet du cinéma (Un film pour l'après-guerre).

Il n'est pas fortuit que quand le "mythe" de la Résistance devient insoutenable, quand ses idéaux libertaires sont écrasés par la décélération de l'élan révolutionnaire dans le monde et par la consolidation sans précédent de l'ordre capitaliste (processus qui en France est marqué par l'institution de la Ve République, en 1958), la philosophie sartrienne de la liberté et de la révolution entre en déclin. Une fois ébranlée la base historique qui avait soutenu "l'offensive existentialiste", d'après l'expression de Simone de Beauvoir, "les espoirs socialistes de 1944" pâlissent et les rêves d'une "génération intellectuelle" se volatilisent - "la Résistance était en miettes", dit Sartre évoquant cette période (Merleau-Ponty vivant). A partir des années 60, comme on le sait, se fait jour l'éclosion d'un type de pensée qui exalte la désagrégation du sujet et conduit à ce que Sartre avait déjà dénoncé, très précocement, comme "idéologies de repli", c'est-à-dire des idéologies qui, fermées dans le discours, aboutissent au. "refus de l'histoire, de la dialectique et de la révolution". Désormais, c'est l'idée de "crise" du marxisme et de la dialectique qui sera au-devant de la scène intellectuelle française. C'est la fin de la vogue sartrienne, c'est la fin d'une époque historique: "l'après-guerre avait fini de finir", écrit Simone de Beauvoir dans La Force des Choses (p. 359). 
Aujourd'hui, tant d'années après le déclin du mouvement "existentialiste", le type de problématique élaborée par Sartre est devenu, si l'on considère le point de vue prépondérant dans le panorama intellectuel français (et pas seulement français), un genre désuet. L'idéologie en vigueur décrète - d'une manière abstraite, puisqu'il s'agit justement d'un décret - la fin de l'ère des révolutions et le début d'une ère post-tout: post-société industrielle, post-lutte de classes, post-économie politique (c'est le cas de Baudrillard), post-marxisme, post-dialectique, post-socialisme, post-modernité (Lyotard, par exemple). Ce discours essentiellement critique de la révolution conduit à ce que l'historien Thompson a diagnostiqué comme un état de "profond conservatisme".

En ce moment marqué par la stérilité des idéologies post-modernes, en ce moment où les chemins de l'histoire semblent si obscurs ("période de décadence" et de "totale désorientation", pour utiliser les expressions avec lesquelles Horkheimer se réfère au capitalisme tardif), ressortent encore plus la fécondité et la vitalité de la pensée sartrienne - cette pensée qui a su faire face aux grands problèmes théoriques et politiques de notre époque. "Ecrivain-chevalier", selon la formule des Mots, Sartre a essayé (quoique plusieurs fois d'une façon ambiguë et paradoxale) de lutter dans deux fronts. D'un côté, contre la "dégénérescence du marxisme", l'autre aspect de la "dégénérescence" de la Révolution. En ce sens, résonnent fortement de nos jours les mots avec lesquels Sartre, en 1956, après avoir condamné l'invasion de la Hongrie, anticipe, pour ainsi dire, le processus social en cours dans l'Europe de l'Est: "C'est avec les royalistes que la Révolution française a fait la République et les républicains; c'est la déstalinisation qui déstalinisera les déstalinisateurs" (Sit., VII, p. 261). Et encore: "la déstalinisation est en cours, c'est la seule politique qui serve, dans le moment présent, le socialisme, la paix, le rapprochement des partis ouvriers" (Sit., VII, p. 307). Cependant, d'un autre côté, Sartre n'a jamais cessé de dénoncer les dangers inhérents aux tentatives de se débarrasser trop rapidement de la perspective du marxisme et, par conséquent, de celle de la lutte de classes et de la révolution. "Le danger de détérioration bureaucratique" d'une révolution est toujours "très grand et constamment présent", dit Sartre en 1970 dans une interview accordée à New Left: "L'idée d'une libération totale et instantanée est une utopie. [...] Mais celui qui en tire argument pour ne pas faire la révolution et pour ne pas lutter dès maintenant pour elle est tout simplement un contre-révolutionnaire" (Sit., IX, p. 129-130). Et dans son dernier grand essai politique, Le socialisme qui venait du froid, Sartre, condamnant l'écrasement, au nom du "socialisme" soviétique, du printemps de Prague - cette expérience qui a fait renaître les "immenses espoirs" et "l'optimisme marxiste" immanents à la Révolution de 1917-, formule ce qu'il considère "la question fondamentale de ce temps": "comment s'unir, liquider les vieilles structures ossifiées, dans quel sens produire les nouvelles pour éviter à la révolution prochaine d'accoucher de Ce socialisme-là?" (Sit., IX, p. 276). Mais n'est-ce pas là la question qui demeure toujours fondamentale pour la gauche à notre époque? Comment éviter à la révolution 
de dégénérer en stalinisme? Et, en même temps, comment éviter que l' 'écroulement du socialisme réel" ne finisse par entraîner avec lui l'aspiration vers un socialisme libertaire et, dans cette mesure, par négliger la lutte pour la justice sociale (problème vital de survivance surtout dans les pays du Tiers Monde)?

L'actualité de cette "question fondamentale" formulée par Sartre se fait vivement sentir en ce moment où l'on tente d'utiliser le processus social en cours dans l'Europe de l'Est (l'escalade du Front national en France et la reprise, dans l'Allemagne de Helmut Khol, du vieux slogan bien connu préconisant une Grande Allemagne en sont de bons exemples) pour éclipser ce qui, comme on le lit dans Qu'est-ce que la littérature?, a été la grande conquête du marxisme: le dévoilement de "la réalité de l'opression et de l'impérialisme capitaliste, de la lutte des classes et de la misère" (Sit., II, p. 246). Et plus loin dans cet essai de 1947, examinant le problème des rapports entre "révolution socialiste" et liberté, Sartre écrit quelque chose qui concerne toute la gauche: "On a souvent prétendu qu'elles [révolution socialiste et liberté] n'étaient pas conciliables: c'est notre affaire de montrer inlassablement qu'elles s'impliquent l'une l'autre" (Sit., II, p. 298).

Aujord'hui, dix années après la mort de Sartre, pensant à la portée de son oeuvre, cette oeuvre qui, justement parce qu'elle a fait des vrais problèmes de notre époque sa matière, aide encore à illuminer la scène du siècle, il nous revient à l'esprit, au sujet de l'auteur lui-même, ses propres mots: "les grands auteurs s'apparentent aux chevaliers errants en ceci que les uns et les autres suscitent des marques passionnées de gratitude" (Les Mots, p. 142).

CREMA, C. D. M. Teoria do Romance e Teoria da Revolução no pensamento de Sartre. Trans/Form/Ação, São Paulo, v. 15, p. 7-37, 1992.

- RESUMO: Reativando o problema da transformação das formas ao longo do itineránio do pensamento de Sartre - da "filosofia pura" à "monografia histórica concreta"-, o presente estudo procura reconstituir alguns momentos do processo de gestação da síntese sartriana entre Filosofia, Romance e Revolução. Teria a "monografia histórica concreta", para Sartre, a função de sucedâneo da "Filosofia Tradicional" e do "Romance Tradicional"? O que parece nortear a trajetória sartriana - de L'Être et le Néant a L'Idiot de la famille - é a busca de uma forma filosófica, literária e histónca que pressupõe, subjacente, uma teoria do romance indissoluvelmente ligada a uma teoria da revolução, mais precisamente, a uma teoria da temporalidade revolucionária que encerra em si mesma a marca decisiva da experiência política da Guerra, da Ocupação e da Resistência.

- UNITERMOS: Teoria do Romance;Teoria da Revolução; "Filosofia Tradicional"; Resistência; "apocalipse". 